Nervenarzt 2017 $\cdot 88: 765-778$

DOI 10.1007/s00115-016-0222-x

Online publiziert: 27. September 2016

(c) Der/die Autor(en) 2016. Dieser Artikel ist

eine Open-Access-Publikation.

CrossMark

\author{
L. Ledochowski' • R. Stark ${ }^{2}$ G. Ruedl' · M. Kopp ${ }^{1}$ \\ 'Institut für Sportwissenschaft, Universität Innsbruck, Innsbruck, Österreich \\ ${ }^{2}$ Kepler Universitätsklinikum, Neuromed Campus, Linz, Österreich
}

\title{
Körperliche Aktivität als therapeutische Intervention bei Depression
}

mit Antidepressiva einen Risikofaktor für kardiovaskuläre Erkrankungen dar [7]. Ein weiteres Problem der pharmakologischen Depressionsbehandlung ist die mangelnde Therapieadhärenz. Es wird angenommen, dass $50 \%$ der psychiatrisch behandelten Patienten und $50 \%$ der hausärztlich versorgten Patienten ihre Therapie selbstständig und vorzeitig abbrechen [8].

Das UK National Institute of Clinical Excellence (NICE; [9]) empfiehlt psychologische und psychotherapeutische $\mathrm{Me}$ thoden zur Behandlung depressiver $\mathrm{Pa}$ tienten. Tatsächlich werden Psychotherapien immer erfolgreicher und häufiger in der Depressionsbehandlung eingesetzt $[10,11]$. Dennoch werden Psychologen und Psychotherapeuten aufgrund der immer noch bestehenden Stigmatisierung häufig ungern von den Patienten aufgesucht [12]; zudem ist der Behandlungsaufwand sowohl aus Sicht der Kostenträger als auch bezogen auf den zu investierenden Zeitaufwand als beträchtlich einzustufen.

Vor dem Hintergrund, dass die soeben genannten Therapiemaßnahmen häufig nur zu einer partiellen Response führen, wird das Interesse an alternativen Therapiemethoden zur Depressionsbehandlung verständlich. Neben den genannten ressourcenintensiven Standardbehandlungsformen Psychopharmakologie und/oder Psychotherapie können sport- und bewegungstherapeutische Maßnahmen eingesetzt werden, da sich gezeigt hat, dass Bewegungsinterventionen Residualsymptomen der Depression oder einem Krankheitsrückfall nach dem Absetzen der Medikamente effektiv entgegenwirken [5]. Bewegungstherapeutische Maßnahmen benötigen weder hohen finanziellen noch technischen Aufwand und sind meist in der Gruppe durchführbar. Therapeutisch begleitetes Ausdauertraining bei Patienten mit depressiven Störungen verbessert nicht nur die Krankheitssymptomatik, sondern spart zusätzlich dem Gesundheitssystem Kosten, indem die stationäre Aufenthaltsdauer verkürzt wird [13].

\section{Ziel der Arbeit}

Im letzten Jahrzehnt haben intensive Forschungsbemühungen zur Erfassung des Potenzials von Bewegungsinterventionen in der Behandlung von Depressionen stattgefunden. Aus diesem Grund erfolgt in der vorliegenden Arbeit eine Zusammenfassung der bestehenden Datenlage $\mathrm{zu}$ dieser Thematik, wobei Bewegung in Anlehnung an Bouchard und Desprès [14] als jegliche körperliche Aktivität definiert wird, welche mit einem erhöhten Energieverbrauch verbunden ist.

Ein Cochrane-Review [15] aus dem Jahr 2013 kam zu dem Schluss, dass - trotz einiger methodischer Mängel in den durchgeführten Forschungsarbeiten - Bewegung im Vergleich zu einer Kontrollbedingung zu einer Verbesserung der depressiven Symptomatik führt. Beim Vergleich der Wirksamkeit von Bewegung mit konventionellen Behandlungsmaßnahmen konnten die Autoren keine signifikanten Unterschiede zwischen den Behandlungsansätzen finden. Ekkekakis [16] unterzog diesen Reviewartikel einer kritischen Prüfung, die sich insbesondere auf Studien bezog, 
welche in ihren Kontrolldesigns ebenfalls Bewegungsinterventionen verwendeten, und konnte nach dem Aufdecken einiger Fehler einen signifikanten Effekt zugunsten von Bewegung verglichen mit konventionellen Therapieansätzen aufzeigen.

Der vorliegende Artikel bietet im deutschsprachigen Raum erstmals einen systematischen Überblick der Fachliteratur zu den Effekten körperlicher Aktivität auf die Symptomatik depressiver Patienten. Aufbauend auf die Übersichtsarbeiten von Rimer et al. [17] und Cooney et al. [15] wird versucht, die seither intensiven Forschungsaktivitäten auf diesem Gebiet zu integrieren.

Neben den Ergebnissen der Zielvariablen (Affekt, Stimmung, Wohlbefinden, depressive Symptomatik) werden die Studiendesigns, Art und Ausmaß der Bewegungseinheit, sowie die verwendeten Erhebungsinventare tabellarisch dargestellt.

Damit sollen Anregungen für den Einsatz bewegungsbezogener Interventionen in der Behandlung von Depressionen und Denkanstöße für die zukünftige Forschung auf diesem Gebiet gegeben werden.

\section{Methode: Suchstrategien und Einschlusskriterien}

Unter Berücksichtigung der Schlüsselwörter („exercise“, „physical activity“, „bipolar disorder", „affective disorder", "depression") wurden die Datenbanken Pubmed (1980 bis März 2016), Web of Science (1980 bis März 2016), EMBASE (1980 bis März 2016), MEDLINE (1950 bis März 2016) und PsycInfo (1980 bis März 2016), durchsucht. Zudem wurden Überblicksartikel und Sekundärliteratur berücksichtigt.

In diese Analyse wurden nur Studien integriert, die den Effekt körperlicher Aktivität bei erwachsenen Personen mit depressiver Störung im Vergleich zu einer Kontrollbedingung (Ruhebedingung, Placebobedingung, pharmakologische Behandlung, psychologische Behandlung, andere Aktivität) untersuchten. Zudem wurde ein kontrolliert randomisiertes Studiendesign (Zuteilung der Probanden zu den Interventions- bzw.
Kontrollgruppen erfolgte unter Verwendung eines Zufallsmechanismus) vorausgesetzt.

\section{Ergebnisse}

Insgesamt wurden 48 Artikel anhand von Titel, Abstract oder Gesamtartikel gefunden ( $\bullet$ Tab. 1). Mit einer randomisierten und kontrollierten Studie untersuchten davon 24 Studien (Studien: $[1,10$, 11, 17-19, 21, 22, 29, 31-36, 39-45, 47, 48]) die Effekte körperlicher Aktivität bei depressiven Patienten im Vergleich zu einer Ruhebedingung (Warteliste, alternative Zuwendung, herkömmliche Behandlung, Kontrollgruppe), 5 (Studien: [14-16, 20, 23]) im Vergleich zu einer psychologischen/psychotherapeutischen Intervention, 9 (Studien: [2-6, 26, 27, 37, 46]) im Vergleich zu pharmakologischen Behandlungen und 6 (Studien: [10, 25, $30,32,38,40])$ im Vergleich zu alternativen Behandlungen (Entspannungstechniken, Lichttherapie ...). 13 (Studien: $[2,7-9,11-13,24,25,28,43,45,47])$ Studien untersuchten die Auswirkungen von unterschiedlichen Bewegungsintensitäten und Modalitäten auf die Symptomatik depressiver Patienten.

Die Auswirkungen einer Bewegungsintervention auf die affektive Befindlichkeit und die Symptomatik depressiver Patienten wurden mit unterschiedlichen Inventaren untersucht. Am häufigsten kamen dabei das Beck Depression Inventory [18] und die Hamilton Rating Scale for Depression [19] zum Einsatz.

Insgesamt $34[2,7-18,20,21,24$, 26-29, 31-35, 37, 39-44, 46, 48] der 48 in den Literaturüberblick aufgenommenen Studien berichteten von signifikant positiven Auswirkungen der Bewegungsinterventionen auf die depressive Symptomatik und affektive Befindlichkeit der Patienten. 5 Studien $[4-6,23,47]$ berichteten von einer tendenziell reduzierten depressiven Symptomatik infolge der Bewegungsintervention, während 9 Studien $[1,3,19,22,25,30,36,38,45]$ keinen positiven Einfluss von Bewegung auf die depressive Symptomatik und affektive Befindlichkeit feststellen konnten.

Um einen genaueren Überblick über die Auswirkungen der Bewegungsintervention der analysierten Studien geben zu können, erfolgt eine deskriptive Bewertung der Ergebnisse geordnet nach den Vergleichsbedingungen (• Tab. 1).

\section{Vergleich mit einer Ruhe- bedingung}

In 18 (Studien: $[10,11,17,18,21,29$, 31-35, 39-44, 48]) der 24 Studien, welche die Effekte körperlicher Aktivität bei depressiven Patienten im Vergleich zu einer Ruhebedingung untersuchten, zeigte sich ein signifikanter Unterschied zwischen den Patienten, welche an einem Bewegungsprogramm teilgenommen hatten, und den Patienten in den Kontrollgruppen. Die Bewegungsinterventionen wirkten sich positiv auf die depressive Symptomatik und affektive Befindlichkeit der untersuchten Patienten aus.

\section{Vergleich mit psycho- therapeutischen Methoden}

Bei einem Vergleich von Bewegungsinterventionen mit psychotherapeutischen Methoden zur Verminderung der depressiven Symptomatik konnte in einer (Studie [20]) von 5 Untersuchungen gezeigt werden, dass Bewegung eher zu einer stärkeren Verminderung der depressiven Symptomatik führt als Psychotherapie. In 4 weiteren Untersuchungen (Studien: [14-16, 23]) zeigte sich, dass sowohl Bewegungsinterventionen als auch kognitive Therapien, sowie eine Kombination dieser beiden Behandlungsansätze zu einer Reduktion der depressiven Symptomatik und zu einer Steigerung der affektiven Befindlichkeit führen.

\section{Vergleich mit psycho- pharmakologischen Maßnahmen}

Beim Vergleich der Wirksamkeit von Bewegung mit der Wirksamkeit psychopharmakologischer Behandlungsmaßnahmen konnten 4 Untersuchungen (Studien: [3-6]) keine signifikanten Unterschiede zwischen diesen Behandlungsansätzen finden. Hingegen zeigten 4 Untersuchungen (Studien: [2, 26, 27, 37]), dass eine kombinierte Therapie aus Bewegung und Psychopharmaka eine bessere Wirkung erzielt als eine rein medikamentöse Behandlung. Eine Studie 
(Studie: [46]) konnte eine signifikante Reduktion der depressiven Symptomatik infolge der Bewegungsintervention finden, jedoch nicht infolge einer medikamentösen Therapie.

\section{Vergleich mit alternativen Behandlungsverfahren}

Bei einem Vergleich der Auswirkungen von Bewegung mit den Auswirkungen alternativer Behandlungsverfahren zeigte sich, dass Ausdauertraining ähnlich positive Effekte auf die affektive Befindlichkeit depressiver Patienten hat wie ein Training zur besseren Körperwahrnehmung (Studie: [10]), Lichttherapie (Studie: [38]) oder Yoga (Studie: [40]). Eine Studie konnte keine signifikante Verminderung der depressiven Symptomatik infolge der Bewegungs- bzw. Entspannungsintervention feststellen (Studie: [25]). Jedoch konnte in dieser Untersuchung ein positiver Effekt regelmäßigen, anstrengenden Zirkeltrainings auf die Arbeitsfähigkeit der untersuchten Patienten gezeigt werden. Dass die Stabilisierung des Schlaf-Wach-Rhythmus in Kombination mit einer Lichttherapie eine effektivere Methode zur Reduzierung der depressiven Symptomatik und Verbesserung der affektiven Befindlichkeit ist als eine tägliche Bewegungseinheit konnte in einer Studie [30] gezeigt werden. Beim Vergleich der Wirksamkeit einer Bewegungsintervention mit einem Muskelrelaxationsprogramm wurde eine Reduktion der depressiven Symptomatik infolge der Bewegungsintervention festgestellt (Studie: [32]).

\section{Wirkung von Bewegungs- intensitäten und -modalitäten}

Insgesamt 10 (Studien: [2, 7-9, 11-13, 24, 28, 43]) der Studien, welche die Auswirkungen von unterschiedlichen Bewegungsintensitäten und -modalitäten auf die Symptomatik depressiver Patienten untersuchten, konnten im Vergleich zur Ausgangserhebung eine Verminderung der depressiven Symptomatik infolge der Bewegungsintervention aufzeigen. In 2 $[2,43]$ Studien wurde festgestellt, dass Bewegungsprogramme hoher Intensität eher zu einer Verminderung depressi-

Nervenarzt 2017 $\cdot 88: 765-778 \quad$ DOI 10.1007/s00115-016-0222-x

(c) Der/die Autor(en) 2016. Dieser Artikel ist eine Open-Access-Publikation.

\section{Ledochowski · R. Stark · G. Ruedl · M. Kopp}

\section{Körperliche Aktivität als therapeutische Intervention bei Depression}

\section{Zusammenfassung}

Ziel. Ziel der Arbeit ist die Darstellung des aktuellen Forschungsstandes zu körperlicher Aktivität als therapeutische Intervention bei Patienten mit Depressionen.

Methodik. Es wurde eine systematische Bewertung von im Zeitraum 1980 bis März 2016 veröffentlichten Fachartikeln, die den Einfluss einer Bewegungsintervention im Vergleich zu einer Kontrollbedingung in der Behandlung von Depressionen untersuchen, durchgeführt. Neben einer Beschreibung der Studiendesigns werden die Ergebnisse zu den verwendeten Zielvariablen depressive Symptomatik (DS) und affektive Befindlichkeit (AB), Art und Ausmaß der Bewegungsintervention und verwendete Erhebungsinventare tabellarisch dargestellt. Ergebnisse. Insgesamt 34 der 48 in den Literaturüberblick aufgenommenen Studien berichten von signifikant positiven Auswirkungen von Bewegungsinterventionen auf die DS und die AB. 5 Studien berichten von einer tendenziell reduzierten DS infolge der Bewegungsintervention. 9 Studien konnten keinen positiven Einfluss von Bewegung auf die DS und die $A B$ feststellen.

Diskussion. Dieser Übersichtssartikel zeigt, dass körperliche Aktivität zu einer Verminderung der Symptomatik und einer Steigerung der affektiven Befindlichkeit bei Patienten mit depressiven Erkrankungen führt. Daher sollten am aktuellen Trainingszustand depressiver Patienten orientierte moderate Bewegungsinterventionen einen Behandlungsbaustein im Rahmen eines mehrdimensionalen therapeutischen Zuganges darstellen.

\section{Schlüsselwörter}

Depression - Affektive Befindlichkeit . Bewegung · Körperliche Aktivität . Evaluierungsstudien

\section{Physical activity as therapeutic intervention for depression}

Abstract

Aim. This article gives a conspectus of the present state of research on the efficiency of exercise as a treatment for patients suffering from depression.

Methods. A systematic review of articles published between December 1980 and March 2016 was carried out. The review focused on studies that examined the effects of exercise compared to control conditions in the treatment of depression. Extracted and analyzed information from the articles included details about participants, characteristics of exercise and control conditions, assessments, study design and outcomes.

Results. A total of 34 of the 48 studies included in the literature search reported a significant reduction of depressive symptoms due to exercise interventions. There was a trend to reduced depressive symptoms following the exercise interventions in five studies. In nine studies no positive impact of exercise on depression and affective wellbeing could be detected.

Discussion. This review article shows that physical activity decreases depressive symptoms and increases affective wellbeing in patients with depressive diseases; therefore, exercise should be recommended as a component of depression treatment within the framework of a multi-dimensional approach.

\section{Keywords}

Depression · Affective well-being · Exercise . Physical activity · Evaluation studies ver Symptomatik führen als solche geringerer Intensität. In einer Untersuchung [24] wurde belegt, dass zügiges Gehen am Laufband depressive Symptome eher verringert als sanftes Stretching.

\section{Diskussion}

Dieser Überblicksartikel zeigt unter Berücksichtigung jüngster experimenteller Daten, dass körperliche Aktivität zu einer Verminderung der Symptomatik und einer Steigerung der affektiven Befindlichkeit bei Patienten mit depressiven Erkrankungen führen kann. Dieser Effekt 


\section{Übersichten}

Tab. 1: Übersicht über die eingeschlossenen kontrollierten randomisierten klinischen Studien

\begin{tabular}{|c|c|c|c|c|c|}
\hline & Studie & $\begin{array}{l}\text { Beschreibung der Stich- } \\
\text { probe }\end{array}$ & Interventionen & Erhebungen & Ergebnisse \\
\hline 1 & Baxter [44-46] & $\begin{array}{l}361 \text { PatientInnen; Diagno- } \\
\text { se: depressive Episode } \\
\text { (bestätigt durch Clinical In- } \\
\text { terview Schedule-Revised, } \\
\text { BDI) Alter: 18-69 Jahre }\end{array}$ & $\begin{array}{l}\text { 1. Gruppe: herkömmliche Behandlung } \\
\text { durch den praktischen Arzt } \\
\text { 2. Gruppe: herkömmliche Behandlung } \\
\text { durch den praktischen Arzt und gemeinsa- } \\
\text { me körperliche Aktivität über } 8 \text { Monate }\end{array}$ & $\begin{array}{l}\text { BDI, SF-12 (Sub- } \\
\text { skalen mentale } \\
\text { und physische } \\
\text { Gesundheit) }\end{array}$ & $\begin{array}{l}\text { Keine Hinweise auf eine } \\
\text { Stimmungsverbesserung in } \\
\text { der 2. Gruppe verglichen mit } \\
\text { der } 1 \text {. Gruppe }\end{array}$ \\
\hline 2 & Belvederi [47] & $\begin{array}{l}121 \text { PatientInnen in der } \\
\text { Erstversorgung mit majo- } \\
\text { rer Depression (HAM-D- } \\
\text { Gesamtscore } \geq 18 \text { ); Alter: } \\
65-85 \text { Jahre }\end{array}$ & $\begin{array}{l}\text { 1. Gruppe Einnahme von Psychopharmaka } \\
\text { (Sertralin - Standarddosis; } n=42 \text { ) } \\
\text { 2. Gruppe Einnahme von Psychophar- } \\
\text { maka (Sertralin - Standarddosis) plus } \\
\text { Bewegungsprogramm geringer Intensität } \\
\text { ( } n=37 \text { ) } \\
\text { 3. Gruppe Einnahme von Psychopharmaka } \\
\text { (Sertralin - Standarddosis) plus Bewe- } \\
\text { gungsprogramm hoher Intensität ( } n=42 \text { ) } \\
\text { Interventionsdauer: } 24 \text { Wochen }\end{array}$ & HAM-D & $\begin{array}{l}\text { Verminderung der depressi- } \\
\text { ven Symptomatik am Ende } \\
\text { der Studie: } \\
\text { Bei } 45 \% \text { der Patientiennen } \\
\text { der Gruppe 1; bei } 73 \% \text { der } \\
\text { Patientiennen der Gruppe 2; } \\
\text { bei } 81 \% \text { der Patientlnnen der } \\
\text { Gruppe } 3 \text { ( } p=0,001 \text { ); schnel- } \\
\text { lere Symptomverminderung } \\
\text { in der Gruppe } 3 \text { verglichen } \\
\text { mit der Gruppe } 1\end{array}$ \\
\hline 3 & $\begin{array}{l}\text { Blumenthal } \\
{[48-50]}\end{array}$ & $\begin{array}{l}\text { Freiwillige; die über Me- } \\
\text { dien rekrutiert wurden; } \\
\text { durchschnittliches Alter: } \\
70 \text { Jahre; } 63 \% \text { Frauen; } \\
n=156\end{array}$ & $\begin{array}{l}\text { 1. Gruppe Walken oder Joggen 3-mal } \\
\text { wöchentlich ( } n=53 \text { ) } \\
\text { 2. Gruppe Einnahme von Psychopharmaka } \\
\text { (Sertralin - Standarddosis; } n=48 \text { ) } \\
\text { 3. Kombination aus Walken/Joggen und } \\
\text { Sertralin ( } n=55 \text { ) } \\
\text { Interventionsdauer: } 16 \text { Wochen } \\
\text { Bewegungsintensität: } 70-85 \% \text { der Ziel- } \\
\text { herzfrequenz }\end{array}$ & HAM-D; BDI & $\begin{array}{l}\text { Kein statistisch signifikan- } \\
\text { ter Unterschied (HAM-D-, } \\
\text { BDI-Scores) zwischen den } \\
\text { unterschiedlichen Gruppen } \\
\text { nach } 16 \text { Behandlungswochen }\end{array}$ \\
\hline 4 & $\begin{array}{l}\text { Blumenthal } \\
{[51,52]}\end{array}$ & $\begin{array}{l}202 \text { Patientlnnen; Diagno- } \\
\text { se: majore Depression; die } \\
\text { über Medien rekrutiert } \\
\text { wurden; durchschnittli- } \\
\text { ches Alter: } 52 \text { Jahre; } 76 \% \\
\text { Frauen }\end{array}$ & $\begin{array}{l}\text { 1. Gruppe Walken/Joggen selbstständig zu } \\
\text { Hause ausgeführt }(n=53) \\
\text { 2. Angeleitetes Walken/Joggen in der } \\
\text { Gruppe }(n=51) \\
\text { 3. Einnahme von Psychopharmaka } \\
\text { (Sertralin - Standarddosis; } n=49) \\
\text { 4. Placebo ( } n=49) \\
\text { Interventionsdauer: } 16 \text { Wochen }\end{array}$ & HAM-D & $\begin{array}{l}\text { Die Effekte körperlicher Ak- } \\
\text { tivität scheinen vergleichbar } \\
\text { mit den Effekten von Anti- } \\
\text { depressiva. Gruppen 1-3 } \\
\text { erzielten bessere Ergebnisse } \\
\text { als Gruppe } 4\end{array}$ \\
\hline 5 & $\begin{array}{l}\text { Blumenthal } \\
\text { [51-53] }\end{array}$ & $\begin{array}{l}\text { Personen über } 35 \text { Jahre, } \\
\text { mit dokumentierter koro- } \\
\text { narer Herzkrankheit und } \\
\text { depressiven Symptomen } \\
(n=101)\end{array}$ & $\begin{array}{l}\text { 1. Gruppe Walken/Joggen/Laufen am } \\
\text { Laufband ( } 65 \% \text { Männer; } n=37 \text { ) } \\
\text { 2. Einnahme von Psychopharmaka } \\
\text { (Sertralin; } 63 \% \text { Männer; } n=40 \text { ) } \\
\text { 3. Placebo ( } 83 \% \text { Männer; } n=24)\end{array}$ & HAM-D & $\begin{array}{l}\text { Reduzierte depressive Sym- } \\
\text { ptomatik in den Gruppen } \\
1 \text { und } 2 \text { verglichen mit der } \\
\text { Gruppe } 3\end{array}$ \\
\hline 6 & Brenes [54] & $\begin{array}{l}\text { Personen über } 65 \text { Jahre } \\
\text { aus Seniorenheimen mit } \\
\text { milder Depression; } 62 \% \\
\text { Frauen; } n=37\end{array}$ & $\begin{array}{l}\text { 1. Gruppe angeleitetes Ausdauer- und } \\
\text { Krafttraining in der Gruppe ( } 60 \text { min, 3-mal } \\
\text { wöchentlich, über } 16 \text { Wochen; } n=14) \\
\text { 2. Einnahme von Psychopharmaka } \\
\text { (Sertralin; } n=11 \text { ) } \\
\text { 3. Kontrollgruppe }\end{array}$ & HAM-D & $\begin{array}{l}\text { Tendenziell reduzierte de- } \\
\text { pressive Symptomatik in den } \\
\text { Gruppen } 1 \text { und } 2 \text { verglichen } \\
\text { mit der Gruppe } 3\end{array}$ \\
\hline 7 & Callaghan [55] & $\begin{array}{l}\text { Frauen, die aufgrund von } \\
\text { Depressionen behan- } \\
\text { delt wurden, im Alter von } \\
45-65 \text { Jahre; } n=38\end{array}$ & $\begin{array}{l}\text { 1. Gruppe: } 12 \text { Einheiten am Laufband } \\
\text { (3-mal wöchentlich) mit selbstgewählter } \\
\text { Geschwindigkeit }(n=19) \\
\text { 2. Gruppe: } 12 \text { Einheiten am Laufband } \\
\text { (3-mal wöchentlich) mit vorgegebener } \\
\text { Geschwindigkeit }(n=19)\end{array}$ & $\begin{array}{l}\text { BDI, RSES, GHQ-12, } \\
\text { QLDS, MDSPSS, } \\
\text { SF12 }\end{array}$ & $\begin{array}{l}\text { Körperliche Aktivität mit } \\
\text { selbstgewählter Geschwin- } \\
\text { digkeit wirkt sich günstig auf } \\
\text { psychologische physiologi- } \\
\text { sche und soziale Parameter } \\
\text { bei depressiven Frauen aus }\end{array}$ \\
\hline 8 & Chu [56] & $\begin{array}{l}\text { Personen mit minorer } \\
\text { und majorer Depression; } \\
\text { durchschnittliches Alter: } \\
26,4 \text { Jahre; } 100 \% \text { Frauen; } \\
n=38\end{array}$ & $\begin{array}{l}\text { 1. Gruppe: bis zu } 5 \text { Ausdauertrainings- } \\
\text { einheiten ( } 1 \text { davon supervidiert) hoher } \\
\text { Intensität pro Woche }(n=15) \\
\text { 2. Gruppe: bis zu } 5 \text { Ausdauertrainingsein- } \\
\text { heiten ( } 1 \text { davon supervidiert) niedriger } \\
\text { Intensität pro Woche }(n=11) \\
\text { 3. Gruppe } 30 \text { min Stretching in der Gruppe } \\
\text { 1-mal wöchentlich }\end{array}$ & $\mathrm{BDI}$ & $\begin{array}{l}\text { Reduzierte depressive Symp- } \\
\text { tomatik in allen } 3 \text { Gruppen }\end{array}$ \\
\hline
\end{tabular}


Tab. 1: Übersicht über die eingeschlossenen kontrollierten randomisierten klinischen Studien (Fortsetzung)

\begin{tabular}{|c|c|c|c|c|c|}
\hline & Studie & $\begin{array}{l}\text { Beschreibung der Stich- } \\
\text { probe }\end{array}$ & Interventionen & Erhebungen & Ergebnisse \\
\hline 9 & Craft $[57,58]$ & $\begin{array}{l}\text { Alter: } 18-55 \text { Jahre; } 32 \\
\text { PatientInnen; Diagnose: } \\
\text { majore Depression, Dys- } \\
\text { thymie oder depressive } \\
\text { Störung }\end{array}$ & $\begin{array}{l}\text { 1. Gruppe: } 2 \text {-mal wöchentlich supervi- } \\
\text { diertes Walken im Medizinzentrum über } 4 \\
\text { Wochen; anschließend } 8 \text { Wochen selbst- } \\
\text { ständiges Training } \\
\text { 2. Gruppe: } 1 \text { supervidierte Walkingeinheit; } \\
\text { anschließend } 12 \text { Wochen selbstständiges } \\
\text { Training }\end{array}$ & BDI & $\begin{array}{l}\text { Reduzierte depressive Symp- } \\
\text { tomatik in beiden Gruppen; } \\
\text { kein statistisch signifikan- } \\
\text { ter Unterschied zwischen } \\
\text { Gruppe } 1 \text { und } 2\end{array}$ \\
\hline 10 & $\begin{array}{l}\text { Danielsson } \\
\text { [59] }\end{array}$ & $\begin{array}{l}62 \text { Erwachsene; Diagno- } \\
\text { se: majore Depression; } \\
\text { erhalten Psychopharmaka }\end{array}$ & $\begin{array}{l}\text { 1. Gruppe: Ausdauertraining über } 10 \text { Wo- } \\
\text { chen } \\
\text { 2. Gruppe: Therapie zur Körperwahrneh- } \\
\text { mung } \\
\text { 3. Gruppe: einmaliges Sitzung mit der } \\
\text { Empfehlung zu körperlicher Aktivität }\end{array}$ & $\begin{array}{l}\text { MADRS; Selbst- } \\
\text { einschätzung von } \\
\text { Depressivität und } \\
\text { Ängstlichkeit }\end{array}$ & $\begin{array}{l}\text { Verbesserung des MADRS } \\
\text { Scores in den Gruppen } 1 \text { und } \\
\text { 2; positive Effekte der Körper- } \\
\text { wahrnehmungstherapie auf } \\
\text { die Selbsteinschätzung von } \\
\text { Depressivität }\end{array}$ \\
\hline 11 & Doyne $[60,61]$ & $\begin{array}{l}\text { Durchschnittliches Alter: } \\
28,5 \text { Jahre; } 100 \% \text { Frauen; } \\
n=40\end{array}$ & $\begin{array}{l}\text { 1. Gruppe: supervidiertes Laufen oder } \\
\text { Gehen 4-mal wöchentlich über } 8 \text { Wochen } \\
\text { 2. Gruppe: supervidiertes Krafttraining } \\
\text { 4-mal wöchentlich } \\
\text { 3. Kontrollgruppe }\end{array}$ & $\begin{array}{l}\text { HAM-D, BDI; Lu- } \\
\text { bin's Depression } \\
\text { Adjective List }\end{array}$ & $\begin{array}{l}\text { Positive Auswirkungen auf } \\
\text { das Selbstkonzept in den } \\
\text { Gruppen } 1 \text { und 2; keine dies- } \\
\text { bezüglichen Unterschiede } \\
\text { zwischen } 1 \text { und } 2\end{array}$ \\
\hline 12 & Dunn $[62,63]$ & $\begin{array}{l}\text { Frauen und Männer im } \\
\text { Alter von } 20-45 \text { Jahren; } \\
\text { durchschnittliches Alter: } \\
35,9 \text { Jahre; } 75 \% \text { Frauen; } \\
n=80\end{array}$ & $\begin{array}{l}4 \text { unterschiedliche Ausdauertrainings- } \\
\text { programme, die sich hinsichtlich Ener- } \\
\text { gieverbrauch (7,0 kcal } / \mathrm{kg} / \text { Woche oder } \\
17,5 \mathrm{kcal} / \mathrm{kg} / \text { Woche) und Häufigkeit (3- } \\
\text { oder } 5 \text {-mal wöchentlich) voneinander } \\
\text { unterscheiden } \\
\text { 1. Gruppe: } 17,5 \mathrm{kcal} / \mathrm{kg} / \text { Woche - } 3 / \text { Woche } \\
\text { ( } n=17) \\
\text { 2. Gruppe: } 17,5 \mathrm{kcal} / \mathrm{kg} / \text { Woche - } 5 / \text { Woche } \\
(n=16) \\
\text { 3. Gruppe: } 7,5 \mathrm{kcal} / \mathrm{kg} / \text { Woche - } 3 / \text { Woche } \\
(n=16) \\
\text { 4. Gruppe: } 7,5 \mathrm{kcal} / \mathrm{kg} / \text { Woche - } 5 / \text { Woche } \\
\text { ( } n=18) \\
\text { 5. Kontrollgruppe mit 3-mal wöchentlich } \\
\text { Bewegung (Laufband, Ergometer) ( } n=13 \text { ) }\end{array}$ & HAM-D & $\begin{array}{l}\text { Reduzierte depressive Sym- } \\
\text { ptomatik in den Gruppen } 1 \\
\text { und } 2 \\
\text { Effekte in den Gruppen } 3 \text { und } \\
4 \text { sind vergleichbar mit denen } \\
\text { der Kontrollgruppe }\end{array}$ \\
\hline 13 & Foley [64] & $\begin{array}{l}\text { Personen mit majorer } \\
\text { Depression; Alter: von } \\
\text { 18-55 Jahre } \\
n=23\end{array}$ & $\begin{array}{l}\text { 1. Gruppe Ausdauertraining moderater } \\
\text { Intensität ( } 30-40 \text { min pro Einheit) } \\
\text { 2. Gruppe Stretching geringer Intensität } \\
\text { 12 Wochen Programm mit } 3 \text { supervidier- } \\
\text { ten Einheiten wöchentlich }\end{array}$ & BDI; MADR & $\begin{array}{l}\text { Reduzierte depressive Symp- } \\
\text { tomatik in beiden Gruppen }\end{array}$ \\
\hline 14 & Fremont [65] & $n=61$ & $\begin{array}{l}\text { 1. Gruppe: supervidiertes Laufen in der } \\
\text { Kleingruppe (6-8 Personen) } 3 \text { mal wö- } \\
\text { chentlich über } 10 \text { Wochen }(n=15) \\
\text { 2. Gruppe: kognitive Therapie ( } 10 \text { Einzel- } \\
\text { stunden) ( } n=16) \\
\text { 3. Gruppe: Kombination aus Laufen und } \\
\text { kognitiver Therapie über } 10 \text { Wochen } \\
(n=18)\end{array}$ & BDI & $\begin{array}{l}\text { Reduzierte depressive Sym- } \\
\text { ptomatik in allen drei Grup- } \\
\text { pen; keine diesbezüglich } \\
\text { statistisch signifikanten Un- } \\
\text { terschiede zwischen den } \\
\text { einzelnen Gruppen }\end{array}$ \\
\hline 15 & Gary [66] & $\begin{array}{l}\text { Depressive Personen mit } \\
\text { Herzfehler (NYHA-Klassifi- } \\
\text { kation II- III); } 42 \% \text { Männer; } \\
\text { Alter: } 30-70 \text { Jahre }\end{array}$ & $\begin{array}{l}\text { 1. Gruppe: Bewegungsprogramm für zu } \\
\text { Hause; Probanden sollen mit moderater } \\
\text { Geschwindigkeit 3-mal wöchentlich bis zu } \\
\text { einer Stunde walken ( } 12 \text { Hausbesuche, um } \\
\text { Ausführung des Bewegungsprogrammes } \\
\text { zu kontrollieren) ( } n=29 \text { ) } \\
\text { 2. Gruppe: Bewegungsprogramm für zu } \\
\text { Hause und kognitive Verhaltenstherapie } \\
\text { ( } n=18 \text { ) } \\
\text { 3. Gruppe: nur kognitive Verhaltensthera- } \\
\text { pie ( } n=17) \\
\text { 4. Gruppe: Kontrollgruppe }\end{array}$ & HAM-D & $\begin{array}{l}\text { Reduktion des HAM-D-Scores } \\
\text { wurde in der } 2 \text {. Gruppe be- } \\
\text { obachtet; gefolgt von der } 3 \text {. } \\
\text { Gruppe }\end{array}$ \\
\hline
\end{tabular}




\section{Übersichten}

Tab. 1: Übersicht über die eingeschlossenen kontrollierten randomisierten klinischen Studien (Fortsetzung) Studie Beschreibung der Stich- Interventionen Erhebungen Ergebnisse

\begin{tabular}{|c|c|c|c|c|c|}
\hline & Studie & $\begin{array}{l}\text { Beschreibung der Stich- } \\
\text { probe }\end{array}$ & Interventionen & Erhebungen & Ergebnisse \\
\hline 16 & Hallgren [67] & $\begin{array}{l}257 \text { Männer und } 689 \text { Frau- } \\
\text { en; Alter: } 18-71 \text { Jahre; aus } \\
\text { der Erstversorgung mit } \\
\text { depressiver Symptomatik }\end{array}$ & $\begin{array}{l}\text { 1. Gruppe: Bewegungsprogramm ( } n=317) \\
\text { 2. Gruppe: internetgestützte kognitive } \\
\text { Verhaltenstherapie }(n=317) \\
\text { 3. Gruppe: herkömmliche Behandlung } \\
(n=312) \\
\text { Interventionsdauer: } 12 \text { Wochen }\end{array}$ & $\begin{array}{l}\text { PHQ-9; Mini-Men- } \\
\text { tal State Exam } \\
\text { Score }\end{array}$ & $\begin{array}{l}\text { Weniger depressive Sympto- } \\
\text { me bei den PatienInnen der } \\
\text { Gruppe } 1 \text { und } 2 \text { verglichen } \\
\text { mit der Gruppe } 3\end{array}$ \\
\hline 17 & $\begin{array}{l}\text { Hemat-Far } \\
{[68]}\end{array}$ & $\begin{array}{l}\text { StudentInnen mit Depres- } \\
\text { sionen; Alter: } 18-25 \text { Jahre; } \\
100 \% \text { Frauen; } n=20\end{array}$ & $\begin{array}{l}\text { 1. Gruppe: supervidiertes Laufen } \\
\text { (40-60 min) 3-mal wöchentlich }(n=10) \\
\text { 2. Gruppe: Kontrollgruppe }(n=10)\end{array}$ & $\mathrm{BDI}$ & $\begin{array}{l}\text { Signifikante Reduktion der } \\
\text { depressiven Symptomatik in } \\
\text { der Interventionsgruppe }\end{array}$ \\
\hline 18 & Ho [69] & $\begin{array}{l}52 \text { PatientInnen einer } \\
\text { psychiatrischen Klinik; Dia- } \\
\text { gnose: majore Depression; } \\
\text { Alter: } 18-64 \text { Jahre }\end{array}$ & $\begin{array}{l}\text { 1. Gruppe: } 5 \text { supervidierte } 30 \text {-minütige } \\
\text { aerobe Trainingseinheiten pro Woche über } \\
3 \text { Wochen } \\
\text { 2. Gruppe: Kontrollgruppe }\end{array}$ & MADRS; BDI & $\begin{array}{l}\text { Signifikante Reduktion des } \\
\text { MADRS-Scores in der Inter- } \\
\text { ventionsgruppe }\end{array}$ \\
\hline 19 & $\begin{array}{l}\text { Hoffman [70, } \\
71]\end{array}$ & $\begin{array}{l}\text { Personen mit Schädel- } \\
\text { Hirn-Trauma ( } 6 \text { Monate } \\
\text { bis } 5 \text { Jahre vor Studienteil- } \\
\text { nahme) mit Depressionen; } \\
n=80\end{array}$ & $\begin{array}{l}\text { 1. Gruppe: } 60 \text { min Ausdauertraining im } \\
\text { Fitnessstudio pro Woche und 4-mal } 30 \text { min } \\
\text { Training zu Hause ( } n=40 ; 38 \% \text { Männer) } \\
\text { 2. Kontrollgruppe (ohne Intervention) } \\
\text { ( } n=40 ; 50 \% \text { Männer) }\end{array}$ & BDI & $\begin{array}{l}\text { Keine statisch signifikanten } \\
\text { Unterschiede zwischen den } \\
\text { einzelnen Gruppen }\end{array}$ \\
\hline 20 & Huang [72] & $\begin{array}{l}57 \text { ältere Personen mit } \\
\text { depressiver Symptomatik }\end{array}$ & $\begin{array}{l}\text { 1. Gruppe: supervidiertes Laufen in der } \\
\text { Kleingruppe ( } 6-8 \text { Personen) 3-mal wö- } \\
\text { chentlich über } 10 \text { Wochen } \\
\text { 2. Gruppe: kognitive Therapie ( } 10 \text { Einzel- } \\
\text { stunden) } \\
\text { 3. Gruppe: Kombination aus Laufen und } \\
\text { kognitiver Therapie über } 10 \text { Wochen }\end{array}$ & GDS-15; SF-36 & $\begin{array}{l}\text { Signifikante Reduktion des } \\
\text { GDS-15-Scores in der Be- } \\
\text { wegungsgruppe zu drei } \\
\text { unterschiedlichen Messzeit- } \\
\text { punkten }\end{array}$ \\
\hline 21 & Kerling [73] & $\begin{array}{l}42 \text { stationäre Patientlnnen } \\
\text { mit majorer Depression; } \\
\text { erhalten Psychopharmaka } \\
\text { und kognitive Verhaltens- } \\
\text { therapie }\end{array}$ & $\begin{array}{l}\text { 1. Gruppe: Bewegungsprogramm, 3-mal } \\
\text { wöchentlich über } 6 \text { Wochen } \\
\text { 2. Gruppe: Kontrollgruppe - übliche Be- } \\
\text { handlung }\end{array}$ & MADRS; BDI - 2 & $\begin{array}{l}\text { Reduziertere depressive } \\
\text { Symptomatik in der Gruppe } 1 \\
\text { im Vergleich zur Gruppe } 2\end{array}$ \\
\hline 22 & Kerse [74] & $\begin{array}{l}193 \text { Personen mit de- } \\
\text { pressiven Symptomen; } \\
\text { durchschnittliches Alter: } \\
81 \text { Jahre; } 59 \% \text { Frauen }\end{array}$ & $\begin{array}{l}\text { 1. Gruppe: individualisiertes Bewegungs- } \\
\text { programm über } 6 \text { Monate } \\
\text { 2. Kontrollgruppe }\end{array}$ & GDS-15 & $\begin{array}{l}\text { Keine statisch signifikanten } \\
\text { Unterschiede zwischen den } \\
\text { beiden Gruppen }\end{array}$ \\
\hline 23 & Klein [75] & $\begin{array}{l}\text { Durchschnittliches Alter: } \\
\text { 30,1 Jahre; } 72 \% \text { Frauen; } \\
n=74\end{array}$ & $\begin{array}{l}\text { 1. Gruppe: supervidiertes Laufen 2-mal } \\
\text { wöchentlich ( } n=27) \\
\text { 2. Gruppe: } \text { kognitive Therapie in der Grup- } \\
\text { pe } 2 \text { h 1-mal wöchentlich ( } n=24) \\
\text { 3. Gruppe: Kontrollgruppe, } 1 \text { h Mediation } \\
\text { 2-mal wöchentlich }(n=23) \\
\text { Interventionsdauer: } 12 \text { Wochen }\end{array}$ & $\begin{array}{l}\text { Symptom Check- } \\
\text { list; Target Symp- } \\
\text { toms; Structural } \\
\text { Analysis of Social } \\
\text { Behaviour; So- } \\
\text { cial Adjustment } \\
\text { Self-reported } \\
\text { Questionnaire; } \\
\text { Cornell Medical } \\
\text { Index; Role rating } \\
\text { Questionnaire; } \\
\text { HAM-D; Global } \\
\text { Assessment Scale }\end{array}$ & $\begin{array}{l}\text { Verminderung der depressi- } \\
\text { ven Symptomatik in allen drei } \\
\text { Gruppen; keine diesbezüglich } \\
\text { signifikanten Unterschiede }\end{array}$ \\
\hline 24 & Knubben [13] & $\begin{array}{l}\text { Stationäre PatientInnen } \\
\text { mit majorer Depression; } \\
\text { durchschnittliches Al- } \\
\text { ter:49 Jahre; } 55 \% \text { Frauen; } \\
n=38\end{array}$ & $\begin{array}{l}\text { 1. Gruppe: Gehen am Laufband für } 10 \text { Tage } \\
\text { ( } n=10) \\
\text { 2. Gruppe: Placebo; täglich leichte } \\
\text { Stretchingübungen für } 30 \text { min }\end{array}$ & $\begin{array}{l}\text { Bech-Rafaelsen } \\
\text { Scale; CES-DS }\end{array}$ & $\begin{array}{l}\text { Signifikante Reduktion der } \\
\text { depressiven Symptomatik in } \\
\text { der Interventionsgruppe }\end{array}$ \\
\hline 25 & Krogh [76-78] & $\begin{array}{l}\text { PatientInnen mit majorer } \\
\text { Depression; durchschnitt- } \\
\text { liches Alter:38,9 Jahre; } \\
73,9 \% \text { Frauen; } n=165\end{array}$ & $\begin{array}{l}\text { 1. Gruppe: anstrengendes Zirkeltraining } \\
(n=55) \\
\text { 2. Gruppe: aerobes Training }(n=55) \\
\text { 3. Kontrollgruppe: Entspannung }(n=55) \\
\text { 2-mal wöchentlich; die Intervention er- } \\
\text { streckt sich über } 32 \text { Einheiten in einem } \\
\text { Zeitraum von } 4 \text { Monaten }\end{array}$ & HAM-D & $\begin{array}{l}\text { Keine signifikante Reduktion } \\
\text { der depressiven Symptomatik } \\
\text { in der Interventionsgruppe }\end{array}$ \\
\hline
\end{tabular}


Tab. 1: Übersicht über die eingeschlossenen kontrollierten randomisierten klinischen Studien (Fortsetzung)

\begin{tabular}{|c|c|c|c|c|c|}
\hline & Studie & $\begin{array}{l}\text { Beschreibung der Stich- } \\
\text { probe }\end{array}$ & Interventionen & Erhebungen & Ergebnisse \\
\hline 26 & La Cerda [79] & 82 depressive Frauen & $\begin{array}{l}\text { 1. Gruppe: Pharmakotherapie (Fluoxetine } \\
20 \text { mg) } \\
\text { 2. Gruppe: Pharmakotherapie plus aerobes } \\
\text { Training; 3-mal wöchentlich über } 8 \text { Wo- } \\
\text { chen (bestehend aus Gymnastik, Tanzen } \\
\text { und Walken) }\end{array}$ & $\begin{array}{l}\text { BDI, ICD-10 Guide } \\
\text { for Depression } \\
\text { Diagnosis }\end{array}$ & $\begin{array}{l}\text { Reduktion der depressiven } \\
\text { Symptomatik in der Interven- } \\
\text { tionsgruppe }\end{array}$ \\
\hline 27 & Lavretsky [80] & $\begin{array}{l}73 \text { PatientInnen, die für } \\
\text { mindestens } 4 \text { Wochen mit } \\
\text { Escitalopram behandelt } \\
\text { wurden }\end{array}$ & $\begin{array}{l}\text { 1. Gruppe: Pharmakotherapie und } 2 \mathrm{~h} \text { Tai } \\
\text { Chi pro Woche über } 10 \text { Wochen } \\
\text { 2. Gruppe: Pharmakotherapie und } 2 \mathrm{~h} \\
\text { Gesundheitserziehung pro Woche über } 10 \\
\text { Wochen }\end{array}$ & $\begin{array}{l}\text { HAM-D, Mini-Men- } \\
\text { tal State Exam } \\
\text { Score; SF-36 Physi- } \\
\text { cal Functioning }\end{array}$ & $\begin{array}{l}\text { Signifikante Reduktion der } \\
\text { depressiven Symptomatik in } \\
\text { der } 1 \text {. Gruppe verglichen mit } \\
\text { der } 2 \text {. Gruppe }\end{array}$ \\
\hline 28 & Legrand [81] & $\begin{array}{l}23 \text { Personen mit ausge- } \\
\text { prägter depressiver Symp- } \\
\text { tomatik }\end{array}$ & $\begin{array}{l}\text { 1. Gruppe: aerobe Trainingseinheiten von } \\
\text { geringer Intensität (Kontrollgruppe; } n=7 \text { ) } \\
\text { 2. Gruppe: aerobe Trainingseinheiten von } \\
\text { hoher Intensität }(n=8) \\
\text { 3. Gruppe: aerobe Trainingseinheiten von } \\
\text { hoher Intensität plus gruppenbasierte } \\
\text { Intervention }(n=8)\end{array}$ & $\mathrm{BDI}$ & $\begin{array}{l}\text { Signifikante Reduktion der } \\
\text { depressiven Symptomatik in } \\
\text { den Gruppen } 2 \text { und 3; keine } \\
\text { signifikanten Unterschie- } \\
\text { de diesbezüglich zwischen } \\
\text { Gruppe } 2 \text { und } 3\end{array}$ \\
\hline 29 & Martinsen [82] & $\begin{array}{l}\text { Stationäre Patientlnnen } \\
\text { einer psychiatrischen } \\
\text { Klinik; durchschnittliches } \\
\text { Alter: } 40 \text { Jahre; } n=43\end{array}$ & $\begin{array}{l}\text { 1. Gruppe: einstündiges angeleitetes Aus- } \\
\text { dauertraining } 3-\text { mal wöchentlich über } 9 \\
\text { Wochen }\left(50-70 \% \mathrm{VO}_{2} \max ; n=28\right) \\
\text { 2. Kontrollgruppe mit alternativer Zuwen- } \\
\text { dung }(n=21)\end{array}$ & $\begin{array}{l}\text { BDI, Comprehen- } \\
\text { sive Psycho-Pa- } \\
\text { thological Rating } \\
\text { Scale Depression } \\
\text { Subscale }\end{array}$ & $\begin{array}{l}\text { Signifikante Reduktion der } \\
\text { depressiven Symptomatik in } \\
\text { der Interventionsgruppe }\end{array}$ \\
\hline 30 & Martiny [83] & $\begin{array}{l}75 \text { erwachsene Patientln- } \\
\text { nen mit majorer Depressi- } \\
\text { on; alle pharmakologisch } \\
\text { mit Duloxetine behandelt }\end{array}$ & $\begin{array}{l}\text { 1. Gruppe: Stabilisierung des Wach-Schlaf- } \\
\text { Rhythmus und Lichttherapie }(n=37) \\
\text { 2. Gruppe: tägliche Bewegungseinheit } \\
(n=38) \\
\text { Interventionsdauer: } 9 \text { Wochen }\end{array}$ & HAM-D & $\begin{array}{l}\text { Signifikante Reduktion der } \\
\text { depressiven Symptomatik in } \\
\text { der Gruppe } 1 \text { verglichen mit } \\
\text { der Gruppe } 2\end{array}$ \\
\hline 31 & Mather [84] & $\begin{array}{l}n=86 \text { (59 Frauen, } 27 \text { Män- } \\
\text { ner); durchschnittliches } \\
\text { Alter in der Interventions- } \\
\text { gruppe: } 63,7 \text { Jahre und } \\
66,2 \text { Jahre in der Kontroll- } \\
\text { gruppe }\end{array}$ & $\begin{array}{l}\text { 1. Gruppe: Ausdauer-, Krafttraining und } \\
\text { Stretching in einer Übungsgruppe für } \\
45 \text { min 2-mal wöchentlich über } 10 \text { Wo- } \\
\text { chen ( } n=43) \\
\text { 2. Kontrollgruppe: Gesundheitserzie- } \\
\text { hungsklasse 2-mal wöchentlich über } 10 \\
\text { Wochen }(n=43)\end{array}$ & $\begin{array}{l}\text { HAM-D; GDS-15; } \\
\text { Clinical Global Im- } \\
\text { pression; Patient } \\
\text { Global Impression }\end{array}$ & $\begin{array}{l}\text { Signifikante Reduktion der } \\
\text { depressiven Symptomatik in } \\
\text { der Interventionsgruppe }\end{array}$ \\
\hline 32 & Mc Cann [85] & $\begin{array}{l}\text { Junge depressive Frauen } \\
(n=47)\end{array}$ & $\begin{array}{l}\text { 1. Gruppe: Ausdauertraining in der Grup- } \\
\text { pe: } 60 \text { min Laufen, Joggen oder Tanzen } \\
\text { 2-mal wöchentlich über } 10 \text { Wochen } \\
\text { ( } n=16) \\
\text { 2. Placebogruppe: Muskelrelaxation für } \\
\text { 15-20 min 4-mal wöchentlich über } 10 \\
\text { Wochen ( } n=15) \\
\text { 3. Kontrollgruppe: }(n=16)\end{array}$ & $\mathrm{BDI}$ & $\begin{array}{l}\text { Reduktion der depressiven } \\
\text { Symptomatik in der Gruppe } 1 \\
\text { verglichen mit den Gruppen } \\
2 \text { und } 3\end{array}$ \\
\hline 33 & Mc Neil [86] & $\begin{array}{l}n=30 ; \text { durchschnittliches } \\
\text { Alter: } 72,5 \text { Jahre }\end{array}$ & $\begin{array}{l}\text { 1. Gruppe: vom Untersucher begleitetes } \\
\text { Walken für } 20 \text { min 3-mal wöchentlich über } \\
6 \text { Wochen } \\
\text { 2. Gruppe: soziale Zuwendung durch } \\
\text { Gespräch mit dem Untersucher 2-mal } \\
\text { wöchentlich } \\
\text { 3. Kontrollgruppe: Warteliste }\end{array}$ & $\mathrm{BDI}$ & $\begin{array}{l}\text { Signifikante Reduktion der } \\
\text { depressiven Symptomatik in } \\
\text { den Gruppen } 1 \text { und 2; Ver- } \\
\text { minderung der somatischen } \\
\text { Symptome (BDI) in der Bewe- } \\
\text { gungsgruppe verglichen mit } \\
\text { den beiden anderen Untersu- } \\
\text { chungsgruppen }\end{array}$ \\
\hline 34 & $\begin{array}{l}\text { Mota-Pereira } \\
{[87]}\end{array}$ & $\begin{array}{l}\text { 18- bis } 60 \text {-Jährige (am- } \\
\text { bulant betreut) mit the- } \\
\text { rapieresistenter majorer } \\
\text { Depression; } n=33\end{array}$ & $\begin{array}{l}\text { 1. Gruppe: } 30-45 \text { min moderates Gehen } \\
\text { 5-mal wöchentlich (davon eine Einheit } \\
\text { beaufsichtigt am Laufband; } n=22 ; 57,9 \% \\
\text { Frauen) } \\
\text { 2. Kontrollgruppe: ohne Intervention } \\
\text { ( } n=11 ; 80 \% \text { Frauen) }\end{array}$ & $\begin{array}{l}\text { BDI , HAM-D; Clin- } \\
\text { ical Global Im- } \\
\text { pression; Global } \\
\text { Assessment of } \\
\text { Functioning Scale }\end{array}$ & $\begin{array}{l}\text { Reduktion der depressiven } \\
\text { Symptomatik in der Gruppe } 1 \\
\text { verglichen mit Gruppe } 2\end{array}$ \\
\hline
\end{tabular}


Tab. 1: Übersicht über die eingeschlossenen kontrollierten randomisierten klinischen Studien (Fortsetzung)

\begin{tabular}{|c|c|c|c|c|c|}
\hline & Studie & $\begin{array}{l}\text { Beschreibung der Stich- } \\
\text { probe }\end{array}$ & Interventionen & Erhebungen & Ergebnisse \\
\hline 35 & $\begin{array}{l}\text { Nabkasorn } \\
{[88]}\end{array}$ & $\begin{array}{l}\text { Krankenpflegeschüler } \\
\text { mit Depressionen; Alter: } \\
\text { 18-20 Jahre; } 100 \% \text { Frau- } \\
\text { en; } n=59\end{array}$ & $\begin{array}{l}\text { 1. Gruppe: Joggen für } 50 \text { min; } 5 \text {-mal wö- } \\
\text { chentlich über } 8 \text { Wochen } \\
\text { 2. Kontrollgruppe }\end{array}$ & CES-DS & $\begin{array}{l}\text { Nach dem Joggen signifikan- } \\
\text { te Reduktion der depressiven } \\
\text { Symptomatik; keine Effekte in } \\
\text { der Kontrollgruppe }\end{array}$ \\
\hline 36 & Pfaff [89] & $\begin{array}{l}200 \text { Erwachsene (50 Jahre } \\
\text { oder älter); mit depressiver } \\
\text { Erkrankung; in ärztlicher } \\
\text { Behandlung }\end{array}$ & $\begin{array}{l}\text { 1. Interventionsgruppe: herkömmliche } \\
\text { ärztliche Behandlung plus } 12 \text {-wöchiges } \\
\text { Trainingsprogramm für zu Hause } \\
\text { 2. Kontrollgruppe; herkömmliche ärztliche } \\
\text { Behandlung }\end{array}$ & $\begin{array}{l}\text { Montgomery- } \\
\text { Asberg Depression } \\
\text { Rating } \\
\text { Scale (SIGMA) }\end{array}$ & $\begin{array}{l}\text { Keine Unterschiede zwischen } \\
\text { den beiden Gruppen hin- } \\
\text { sichtlich Ausprägung der } \\
\text { depressiven Symptomatik }\end{array}$ \\
\hline 37 & Pilu $[90,91]$ & $\begin{array}{l}\text { Personen mit majorer De- } \\
\text { pression; Alter: } 40-60 \text { Jah- } \\
\text { re; } 100 \% \text { Frauen; } n=30\end{array}$ & $\begin{array}{l}\text { 1. Gruppe: } 60 \text { min Kräftigungsübungen } \\
\text { 2-mal wöchentlich und pharmakologische } \\
\text { Behandlung ( } n=10) \\
\text { 2. Gruppe: nur pharmakologische Behand- } \\
\text { lung }(n=20)\end{array}$ & HAM-D & $\begin{array}{l}\text { Verbesserung des HAM-D- } \\
\text { Scores in der 1. Gruppe; keine } \\
\text { Verbesserung in der Kontroll- } \\
\text { gruppe }\end{array}$ \\
\hline 38 & Pinchasov [92] & $\begin{array}{l}n=63 ; \text { durchschnittliches } \\
\text { Alter: } 35,2 \text { Jahre; } 100 \% \\
\text { Frauen }\end{array}$ & $\begin{array}{l}\text { 1. Gruppe: täglich } 54 \text { min Radfahren am } \\
\text { Ergometer für } 1 \text { Woche } \\
\text { 2. Gruppe: Lichttherapie }\end{array}$ & HAM-D & $\begin{array}{l}\text { PatientInnen mit saisona- } \\
\text { ler Depression reagierten } \\
\text { sowohl positiv auf Licht als } \\
\text { auch auf Bewegung (keine } \\
\text { signifikanten Unterschie- } \\
\text { de diesbezüglich zwischen } \\
\text { Gruppe } 1 \text { und 2) }\end{array}$ \\
\hline 39 & Schuch [93] & $\begin{array}{l}\text { Stationäre PatientInnen } \\
\text { mit schweren Depressio- } \\
\text { nen; } n=26\end{array}$ & $\begin{array}{l}\text { 1. Gruppe: } 3 \text { Einheiten selbstgewählten } \\
\text { Ausdauertrainings pro Woche }(n=15) \\
\text { 2. Kontrollgruppe: konventionelle Thera- } \\
\text { pie }\end{array}$ & HAM-D & $\begin{array}{l}\text { Signifikante Reduktion der } \\
\text { HAM-D-Werte in der Trai- } \\
\text { ningsgruppe zum Entlas- } \\
\text { sungszeitpunkt }\end{array}$ \\
\hline 40 & Shahidi [94] & $\begin{array}{l}\text { Freiwillige im Alter von } \\
\text { 18-35 Jahren; } 26 \% \text { Frau- } \\
\text { en; } n=150\end{array}$ & $\begin{array}{l}\text { 1. Gruppe: } 10 \text { Einheiten zu je } 30 \text { min ae- } \\
\text { robes Training in der Gruppe (Joggen und } \\
\text { Dehnen; } n=23 \text { ) } \\
\text { 2. Gruppe: "Laughter Yoga“ ( } n=23) \\
\text { 3. Gruppe: Kontrollgruppe }(n=24)\end{array}$ & $\begin{array}{l}\text { GDS; Life satisfacti- } \\
\text { on Scale }\end{array}$ & $\begin{array}{l}\text { Reduktion der depressiven } \\
\text { Symptomatikin den Gruppen } \\
1 \text { und } 2 \text { verglichen mit Grup- } \\
\text { pe } 3 \text {; keine diesbezüglich } \\
\text { signifikanten Unterschiede } \\
\text { zwischen Gruppe } 1 \text { und } 2\end{array}$ \\
\hline 41 & Sims $[95,96]$ & $\begin{array}{l}\text { SchlaganfallpatientInnen } \\
\text { mit psychiatrisch bestätig- } \\
\text { ter Depression; rekrutiert } \\
\text { über Klinikdatenbanken, } \\
\text { Ärzte und Zeitungsanzei- } \\
\text { gen; } n=45 ; \text { durchschnitt- } \\
\text { liches Alter: } 67,13 \text { Jahre; } \\
40 \% \text { Frauen }\end{array}$ & $\begin{array}{l}\text { 1. Gruppe: } 2 \text { Trainingseinheiten hoher } \\
\text { Intensität pro Woche in der Gruppe über } \\
10 \text { Wochen ( } n=23) \\
\text { 2. Gruppe: Kontrollgruppe (Warteliste; } \\
n=22)\end{array}$ & CES-DS & $\begin{array}{l}\text { Die Interventionsgruppe } \\
\text { zeigte niedrigere Depressi- } \\
\text { onsscores zu allen Messzeit- } \\
\text { punkten verglichen mit der } \\
\text { Kontrollgruppe }\end{array}$ \\
\hline 42 & Singh [97] & $\begin{array}{l}\text { Freiwillige im Alter von } \\
61-88 \text { Jahren; } 63 \% \text { Frau- } \\
\text { en; } n=32\end{array}$ & $\begin{array}{l}\text { 1. Gruppe: supervidiertes nicht aerobes } \\
\text { Zirkeltraining 3-mal wöchentlich über } 10 \\
\text { Wochen }(n=17) \\
\text { 2. Gruppe: Kontrollgruppe 2-mal wöchent- } \\
\text { lich Seminare zum Thema Gesundheit } \\
(n=15)\end{array}$ & HAM-D, BDI & $\begin{array}{l}\text { Signifikante Reduktion der } \\
\text { HAM-D- und BDI-Werte in der } \\
\text { Gruppe } 1 \text { verglichen mit der } \\
\text { Gruppe } 2\end{array}$ \\
\hline 43 & Singh [98] & $\begin{array}{l}\text { Durchschnittliches Alter: } \\
69 \text { Jahre; } 55 \% \text { Frauen; } \\
n=60\end{array}$ & $\begin{array}{l}\text { 1. Gruppe: supervidiertes nichtaerobes } \\
\text { Zirkeltraining hoher Intensität } \\
\text { 2. Gruppe: supervidiertes nichtaerobes } \\
\text { Zirkeltraining geringer Intensität } \\
\text { Interventionsdauer: } 3 \text {-mal wöchentlich } \\
\text { über } 8 \text { Wochen } \\
\text { 3. Gruppe: Kontrollgruppe 2-mal wöchent- } \\
\text { lich Seminare zum Thema Gesundheit }\end{array}$ & HAM-D & $\begin{array}{l}50 \% \text { Reduktion der depres- } \\
\text { siven Symptomatik bei } 61 \% \\
\text { der } 1 . \text { Gruppe; } 29 \% \text { der } 2 . \\
\text { Gruppe und } 21 \% \text { der } 3 . \\
\text { Gruppe ( } p=0,03) ; \text { Zirkel- } \\
\text { training hoher Intensität ist } \\
\text { effektiver in der Depressions- } \\
\text { behandlung als Zirkeltraining } \\
\text { geringer Intensität }\end{array}$ \\
\hline 44 & Tsang [99] & $\begin{array}{l}85 \text { PatientInnen; Alter } \\
>65 \text { Jahre }\end{array}$ & $\begin{array}{l}\text { 1. Gruppe: Qigong-Übungen } \\
\text { 2. Gruppe: Lesegruppe (Kontrollgruppe) } \\
\text { Interventionsdauer: } 16 \text { Wochen }\end{array}$ & $\begin{array}{l}\text { GDS-15, Personal } \\
\text { Well Being Index, } \\
\text { Chinese General } \\
\text { Self-efficacy Scale, } \\
\text { GHQ-12 }\end{array}$ & $\begin{array}{l}\text { Stimmungsverbesserung, } \\
\text { erhöhte Selbstwirksamkeit } \\
\text { und erhöhtes Wohlergehen } \\
\text { in der Gruppe } 1\end{array}$ \\
\hline
\end{tabular}




\begin{tabular}{|c|c|c|c|c|c|}
\hline & Studie & $\begin{array}{l}\text { Beschreibung der Stich- } \\
\text { probe }\end{array}$ & Interventionen & Erhebungen & Ergebnisse \\
\hline 45 & Veale [100] & $\begin{array}{l}2 \text { klinische Studien; ins- } \\
\text { gesamt } 124 \text { Probanden; } \\
\text { durchschnittliches Alter: } \\
35,5 \text { Jahre; } 64 \% \text { Frauen; } \\
33 \% \text { erhielten zum Studi- } \\
\text { enzeitpunkt Anti- } \\
\text { depressiva und } 24 \% \text { Ben- } \\
\text { zodiazepine }\end{array}$ & $\begin{array}{l}\text { Studie } 1 \text { ( } n=83 \text { ): } \\
\text { 1. Gruppe: aerobes Training } \\
\text { 2. Gruppe: Kontrollgruppe } \\
\text { Interventionsdauer: } 12 \text { Wochen } \\
\text { Studie } 2(n=41): \\
\text { 1. Gruppe: aerobes Training } \\
\text { 2. Gruppe: körperliche Aktivität geringer } \\
\text { Intensität } \\
\text { Interventionsdauer: } 12 \text { Wochen }\end{array}$ & $\begin{array}{l}\text { CIS, Social Sup- } \\
\text { ports and Stresses } \\
\text { Interview, BDI, } \\
\text { STAI } 13\end{array}$ & $\begin{array}{l}\text { Studie 1: kein signifikan- } \\
\text { ter Unterschied in der } \\
\text { BDI-Ausprägung zwischen } \\
\text { Gruppe } 1 \text { und } 2 \\
\text { Studie 2: kein signifikan- } \\
\text { ter Unterschied in der } \\
\text { BDI-Ausprägung zwischen } \\
\text { Gruppe } 1 \text { und } 2\end{array}$ \\
\hline 46 & Verrusio [101] & $\begin{array}{l}24 \text { Probanden mit majorer } \\
\text { Depression; durchschnitt- } \\
\text { liches Alter: } 75 \text { Jahre; } 13 \\
\text { Frauen }\end{array}$ & $\begin{array}{l}\text { 1. Gruppe: Pharmakotherapie (Paroxetin, } \\
\text { Mirtazapin) } \\
\text { 2. Gruppe: Bewegungsprogramm mit } \\
\text { Musik } 1 \text { h 2-mal wöchentlich } \\
\text { Interventionsdauer: } 6 \text { Monate }\end{array}$ & GDS; HAS & $\begin{array}{l}\text { In der Gruppe } 2 \text { konnte nach } \\
3 \text { und nach } 6 \text { Monaten eine } \\
\text { signifikante Reduktion der } \\
\text { depressiven Symptomatik } \\
\text { beobachtet werden }\end{array}$ \\
\hline 47 & Williams [102] & $\begin{array}{l}45 \text { depressive, demen- } \\
\text { te Altersheimbewohner; } \\
\text { durchschnittliches Al- } \\
\text { ter:87,9 Jahre; } 89 \% \text { Frauen }\end{array}$ & $\begin{array}{l}\text { 1. Gruppe: umfangreiches individuelles } \\
\text { Training (Kräftigungsübungen, Balance- } \\
\text { übungen, 20-minütiges Walken) } \\
\text { 2. Gruppe: supervidiertes Walken (für } \\
30 \text { min) } \\
\text { 3. Gruppe: Kontrollgruppe/ soziale Auf- } \\
\text { merksamkeit (Gespräche mit den Heim- } \\
\text { bewohnern) } \\
\text { Interventionsdauer: } 16 \text { Wochen }\end{array}$ & $\begin{array}{l}\text { CSDD, DMAS, AMS, } \\
\text { OAS }\end{array}$ & $\begin{array}{l}\text { Reduzierte depressive Symp- } \\
\text { tomatik in allen drei Grup- } \\
\text { pen; tendenziell stärker } \\
\text { ausgeprägt in den beiden } \\
\text { Bewegungsgruppen }\end{array}$ \\
\hline 48 & $\begin{array}{l}\text { Zarshenas } \\
{[103]}\end{array}$ & $\begin{array}{l}82 \text { Frauen mit einem } \\
\text { BDI-Gesamtscore }>14 \text {; } \\
\text { durchschnittliches Alter: } \\
26 \text { Jahre }\end{array}$ & $\begin{array}{l}\text { 1. Gruppe: aerobes Training } \\
\text { 2. Gruppe: Kontrollgruppe - Warteliste } \\
\text { Interventionsdauer: } 4 \text { Wochen }\end{array}$ & MDBSRQ, BDI & $\begin{array}{l}\text { Signifikante Reduktion der } \\
\text { BDI-Werte in der Gruppe } 1 \\
\text { verglichen mit der Gruppe } 2 \\
(p<0,5)\end{array}$ \\
\hline \multicolumn{6}{|c|}{$\begin{array}{l}\text { BDI Beck Depression Inventory, SF-12 Health Survey, HAM-D Hamilton Rating Scale for Depression, RSES Rosenberg Self Esteem Scale, GHQ-12 General } \\
\text { Health Questionnaire 12, QLDS Quality of Life in Depression Scale, MDSPSS Multi-Dimensional Scale of Perceived Social Support, MADRS Montgomery } \\
\text { Asberg Rating Scale, PHQ-9 Patient Health Questionnaire, GDS-15 Geriatric Depression Scale, CES-DS Center for Epidemiologic Studies Depression Scale, } \\
\text { SF } 36 \text { Health Survey, STAI } 13 \text { State-Trait anxiety inventory13, CSDD Cornell Scale for Depression in Dementia, DMAS Dementia Mood Assessment Scale, } \\
\text { AMS Alzheimer's Mood Scale, OAS Observed Affect Scale, MDBSRQ Multidimensional Body Self-Relation Questionnaire }\end{array}$} \\
\hline
\end{tabular}

konnte sowohl bei Patienten mit unipolaren als auch bei Patienten mit bipolaren Depressionen beobachtet werden, wobei hervorgehoben werden muss, dass die in diesen Überblicksartikel aufgenommenen Studien vorwiegend unipolar depressive Patienten untersucht haben.

\section{Physiologische und psycho- logische Erklärungsmodelle}

Für die zielgruppenorientierte Entwicklung effektiver Bewegungsprogramme zur Behandlung depressiver Erkrankungen ist eine Erklärung für die experimentell bestätigte Kausalbeziehung von Bewegung und der Verringerung depressiver Symptome wichtig. Es existieren viele physiologische und psychologische Modelle zur Erklärung dieses Wirkmechanismus [20]. Die meisten konnten jedoch bis heute nicht empirisch belegt werden [21].
Neben Erklärungsmöglichkeiten wie der Endorphinhypothese, der Ablenkungshypothese, der Selbstwirksamkeitshypothese oder der Theorie meditativer Bewusstseinszustände, die sich populärwissenschaftlicher Beliebtheit erfreuen, scheinen vor allem die transiente Hypofrontalitätstheorie (THFT), das Dual-Mode-Modell (DMM), das serotonerge System sowie die ,adulte Neurogenese" Aufschluss über dieses Phänomen zu geben.

\section{Hypofrontalitätstheorie}

Die THFT geht davon aus, dass während körperlicher Bewegung spezifische Gehirnareale aktiviert werden. Untersuchungen mittels Positronenemissionstomographie (PET), Elektroenzephalographie (EEG) und Single-Photonen-Emissions-Computertomographie (SPECT) haben gezeigt, dass während Bewegung die motorischen, sensori- schen und autonomen Regionen des Gehirns aktiv sind [22]. Da bestimmte Bewegungsausführungen ein komplexes Zusammenspiel einer Vielzahl von Muskeln voraussetzen, ist für die erfolgreiche Bewegungsausführung eine Art „Rechenleistung“ in den entsprechenden Gehirnarealen notwendig [22]. Das führt $\mathrm{zu}$ einer erhöhten Sauerstoffnachfrage. Da der Blutfluss zum Gehirn und der zerebrale Stoffwechsel aber nicht ansteigen, kommt es während Bewegung zu einer Ressourcenverschiebung [23]. Das heißt, dass vor allem sensorische, motorische und autonome Gehirnregionen versorgt werden, während neuronale Aktivitäten in neutralen Strukturen, die für die Bewegungsausführung unwesentlich sind, gesenkt werden [24]. Dabei werden vor allem Areale im präfrontalen Kortex, die für Kontrollprozesse mit dem Ziel der Top-down-Kontrolle von Kognition 
und Emotion zuständig sind [25, 26], herunterreguliert.

Studien konnten zeigen, dass es möglich ist, auf diese Weise zumindest für den Zeitraum der Bewegungsausführung negativen emotionalen Zuständen zu entkommen [27].

\section{Dual-Mode-Modell}

Im DMM werden physiologische und psychische Mechanismen zur Erklärung der Befindlichkeitssteigerung infolge von Bewegung eingebunden. Dabei werden vor allem die „Dosis-Wirkungs-Beziehung ", ,interindividuelle Unterschiede in der Affektantwort“, und „die Vielfältigkeit der affektiven Antworten" berücksichtigt [28, 29]. Das DMM geht davon aus, dass die „Affektantworten“ auf physische Aktivität durch zwei (sich gegenseitig beeinflussende) Faktoren ausgelöst werden. Ein Faktor wird dabei von „Wahrnehmung", „Kognition“ und „Erkenntnis" gebildet, ein anderer von den „interozeptiven Signalen“ (Innenreizen) über den Zustand des Körpers (z. B. thermische, muskuläre, respiratorische Parameter). Es wird angenommen, dass sich das Verhältnis dieser beiden Faktoren gleichzeitig mit der Belastungsintensität ändert. Bei körperlicher Aktivität geringer und mittlerer Intensität werden die affektiven Antworten von „kognitiven Faktoren" determiniert [28, 29]. Bei körperlicher Aktivität hoher Intensität gewinnen hingegen dazu die „interozeptiven Signale" mehr an Einfluss.

Demnach sind die affektiven Antworten auf Bewegung geringer Intensität vorwiegend positiv, während bei zu intensiver Bewegung vermehrt negative affektive Antworten beobachtet werden konnten $[28,29]$.

\section{Das serotonerge System}

Einen weiteren Erklärungsansatz bietet die Regulation des Serotoninspiegels durch Ausdauertraining [30]. Serotonin ist ein Neurotransmitter, der sowohl das Verhalten als auch die Stimmung eines Individuums beeinflusst. Serotoninmangel kann zur Ausbildung depressiver Symptome führen [31].

Ausgangssubstanz für die Bildung des Serotonins ist die essenzielle Aminosäure Tryptophan. Damit eine ausreichende
Serotoninsynthese im Gehirn erfolgen kann, muss „freies Tryptophan“ die BlutHirn-Schranke passieren. Tryptophan „konkurriert" jedoch mit anderen (neutralen) Aminosäuren, den LNAA (,large neutral amino acids": Valin, Isoleucin und Leucin) um denselben Transporter an der Blut-Hirn-Schranke. LNAA werden durch die Ausschüttung von Insulin in die Muskelzelle aufgenommen [32]. Das hat einen positiven Effekt auf die Tryptophanaufnahme im Gehirn. Die Insulinausschüttung (für den Transport der LNAA in die Arbeitsmuskulatur) wird unter anderem durch körperliche Aktivität hervorgerufen [33].

\section{"Adulte Neurogenese"}

Direkte Effekte von Bewegung auf die Neubildung von Neuronen liefern eine weitere Erklärung für die Befindlichkeitssteigerung infolge körperlicher Aktivität $[30,34]$. Depressionen gehen offenbar mit einem verringerten Hippokampusvolumen einher [35]. Tierexperimentelle Untersuchungen haben gezeigt, dass motorische Aktivität und körperliche Belastung sowohl zu einem Anstieg der regionalen Gehirndurchblutung als auch zu einem Anstieg neurotropher Wachstumsfaktoren führen. Dadurch wird die Vernetzung und Neubildung von Nervenzellen im Hippokampus begünstigt [36].

Mit diesen Theorien kann die positive affektive Antwort auf Bewegung bzw. die Verringerung der depressiven Symptomatik infolge von Bewegung erklärt werden. Dennoch sind weitere Untersuchungen erforderlich, um die anhaltenden Effekte von Bewegung aufdas psychische Wohlbefinden vollständig aufklären zu können. Dabei sollten sowohl biochemische, physiologische als auch psychologische Ansätze integriert werden.

\section{Ausblick}

Aber nicht nur die Ursachenaufklärung des beobachteten Effektes von Bewegung auf die affektive Befindlichkeit und die depressive Symptomatik von Patienten bedarf weiterer Untersuchungen. Bei Betrachtung des aktuellen Forschungsstandes dazu, sollten zukünftig sowohl unmittelbare als auch langzeitige Effekte von Bewegung, sowie die Auswirkungen unterschiedlicher Bewegungsintensitäten und -formen auf die affektive Befindlichkeit und die Symptomatik von Patienten mit depressiven Erkrankungen untersucht werden.

\section{Motivation und Compliance}

Die zunehmende wissenschaftliche Beweislast vom Nutzen körperlicher Aktivität bei depressiven Erkrankungen steht einer niedrigen Motivation und Compliance der Patienten gegenüber. Es ist notwendig, diese durch einen bewegungsarmen Lebensstil zu beschreibende Patientengruppe $[6,37]$ nachhaltig zu mehr körperlicher Bewegung zu motivieren. Es hat sich gezeigt, dass die Compliance psychiatrischer Patienten für die Teilnahme an einem Ausdauertrainingsprogramm durch Techniken der motivierenden Gesprächsführung (z. B. Konzept der Entscheidungsbalance) deutlich erhöht werden konnte [38]. Das tägliche Protokollieren der Aktivitäten und die engmaschige persönliche Betreuung durch den Bewegungstherapeuten [39], sowie Gespräche zu Beginn der Bewegungsintervention über mögliche Hindernisse zur Aufrechterhaltung der körperlichen Aktivität und regelmäßiges Feedback über die erzielten Fortschritte im Lauf des Bewegungsprogramms [37] führten zu einer regelmäßigeren Beteiligung an den Bewegungsprogrammen. Obwohl bisher keine Daten zu der Höhe der tatsächlichen durchschnittlichen Abbruchsquote von Bewegungsinterventionen bei depressiven Patienten vorliegen, sollten sich weitere Untersuchungen damit auseinander setzen, wie eine Reduktion dieser Abbruchsquote erreicht werden kann.

Bisher gibt es widersprüchliche Empfehlungen bezüglich Dauer, Frequenz und Intensität der Bewegungsinterventionen bei depressiven Patienten [40]. Daher wäre es von hohem Interesse, jene „optimale Bewegungsdosis“ ausfindig zu machen, welche eine positive Veränderung der affektiven Befindlichkeit bzw. der depressiven Symptomatik bewirkt. Eine Befragung depressiver Patienten zu den Präferenzen von Bewegung als Behandlungsmethode hat ergeben, dass ein einmal täglich angeleitetes 30 - bis 60minütiges Training (bevorzugt Walken) 
moderater Intensität erwünscht wird [41].

\section{Studiendesigns}

Obwohl in diesen Überblicksartikel auch Studien mit teilweise kleinen Stichproben und nichtstandardisierten Inklusionskriterien aufgenommen wurden, lässt sich aufgrund der verfügbaren Datenlage insgesamt eindeutig ein positiver Effekt von Bewegungsinterventionen auf die affektive Befindlichkeit und die Symptomatik depressiver Patienten feststellen. Zur weiteren Verbesserung der Datenlage sollten zukünftige Studien vor allem mit randomisierten Untersuchungsdesigns und mit homogenen Kontrollgruppen bzw. Kontrollbedingungen arbeiten. Um eine bessere Vergleichbarkeit der Studien zu gewährleisten, sollte eine möglichst einheitliche methodische Vorgangsweise bei der Erfassung der Zielvariablen (affektive Befindlichkeit und depressive Symptomatik) gewählt werden.

\section{Behandlungsempfehlung}

Es ist bekannt, dass depressive Patienten eine deutlich reduzierte Lebenserwartung aufweisen [42]. Durch die Integration körperlicher Aktivität in die Behandlung von Patienten mit Depressionen könnte neben der Verbesserung der krankheitsbedingten Symptomatik auch eine Verbesserung des somatischen Zustandsbildes dieser Patientengruppe durch die Reduktion kardiovaskulärer Risikofaktoren erreicht werden. Das gilt trotz höherer somatischer Komorbidität auch für depressive Patienten im höheren Lebensalter. Für ältere Patienten sollten die Trainingsprogramme Ausdauerund Kräftigungseinheiten beinhalten, sie sollten methodisch gut strukturiert sein und an den Trainingszustand und die individuellen Fähigkeiten der Patienten angepasst werden [43].

Um eine konkrete Behandlungsempfehlung für depressive Patienten entwickeln zu können, sind weitere Forschungsbemühungen notwendig. Insbesondere Informationen zur idealen Bewegungsdauer, -häufigkeit und -intensität sowohl bezüglich unmittelbarer Auswirkungen während und nach einzelnen Bewegungseinheiten als auch bezüglich längerfristiger Bewegungsinterventionen sind dabei von Interesse.

\section{Fazit für die Praxis}

Für die psychiatrische Praxis lässt sich empfehlen, dass am aktuellen Trainingszustand depressiver Patienten orientierte moderate Bewegungsinterventionen auf Basis des aktuellen Forschungsstandes einen Behandlungsbaustein im Rahmen eines mehrdimensionalen therapeutischen Zuganges darstellen sollten.

\section{Korrespondenzadresse}

\section{Dr. L. Ledochowski}

Institut für Sportwissenschaft, Universität Innsbruck

Fürstenweg 185, 6020 Innsbruck, Österreich Larissa.ledochowski@uibk.ac.at

Open access funding provided by University of Innsbruck and Medical University of Innsbruck.

\section{Einhaltung ethischer Richtlinien}

Interessenkonflikt. L. Ledochowski, R. Stark, G. Ruedl und M. Kopp geben an, dass kein Interessenkonflikt besteht.

Dieser Beitrag beinhaltet keine von den Autoren durchgeführten Studien an Menschen oder Tieren.

Open Access. Dieser Artikel wird unter der Creative Commons Namensnennung 4.0 International Lizenz (http://creativecommons.org/licenses/by/4.0/deed. de) veröffentlicht, welche die Nutzung, Vervielfältigung, Bearbeitung, Verbreitung und Wiedergabe in jeglichem Medium und Format erlaubt, sofern Sie den/die ursprünglichen Autor(en) und die Quelle ordnungsgemäßnennen, einen Linkzur Creative Commons Lizenz beifügen und angeben, ob Änderungen vorgenommen wurden.

\section{Literatur}

1. Kail N (2007) Neue Leitlinie: Depressive Erkrankungen. Osterr Arzteztg 5. http://www. aerztezeitung.at/archiv/oeaez-2007/oeaez-510032007/depressive-erkrankungen.html.Zugegriffen: 22.9.2016

2. Arroll B, Elley CR, Fishman T, Goodyear-Smith FA, Kenealy T, Blashki G et al (2009) Antidepressants versus placebo for depression in primary care. Cochrane Database Syst Rev 3:CD007954. doi:10. 1002/14651858.cd007954

3. Fava GA, Ruini C (2002) The sequential approach to relapse prevention in unipolar depression. World Psychiatry 1(1):10-15

4. Dording CM, Mischoulon D, Petersen TJ, Kornbluh R, Gordon J, Nierenberg AA et al (2002) The pharmacologic management of SSRI-induced side effects: a survey of psychiatrists. Ann Clin Psychiatry 14(3):143-147

5. Trivedi MH, Greer TL, Grannemann BD, Church TS, Galper DI, Sunderajan P et al (2006) TREAD: Treatment with Exercise Augmentation for Depression: study rationale and design. Clin Trials 3(3):291-305

6. Kopp M, Fleischhacker WW, Stürz K, RuedI G, Kumnig M, Rumpold G (2011) Poor health behaviour and reduced quality of life of people treated with psychotropic drugs. Hum Psychopharmacol Clin Exp 26(2):161-167

7. Waring WS (2012) Clinical use of antidepressant therapy and associated cardiovascular risk. Drug Healthc Patient Saf 4:93-101

8. Sansone RA, Sansone LA (2012) Antidepressant adherence: are patients taking their medications? Innov Clin Neurosci 9(5-6):41-46

9. National Institute for Health and Clinical Excellence (2009) Depression: the treatment and management of depression in adults. www.nice.org.uk/ CG90.Zugegriffen: 14. März2014

10. Ambresin G, Despland J, Preisig M, de Roten $Y$ (2012) Efficacy of an adjunctive brief psychodynamic psychotherapy to usual inpatient treatment of depression: rationale and design of a randomized controlled trial. BMC Psychiatry 12:182

11. Brakemeier E, Frase L (2012) Interpersonal psychotherapy (IPT) in major depressive disorder. Eur Arch Psychiatry Clin Neurosci 262(Suppl 2):117-121

12. Schomerus $G$, Matschinger $H$, Angermeyer MC (2014) Causal beliefs of the public and social acceptance of persons with mental illness: a comparative analysis of schizophrenia, depression and alcohol dependence. Psychol Med 44(2):303-314

13. Knubben K, Reischies FM, Adli M, Schlattmann P, Bauer M, Dimeo F (2007) A randomised, controlled study on the effects of a short-term endurance training programme in patients with major depression. BrJSports Med 41(1):29-33

14. Bouchard C, Després JP (1995) Physical activity and health: atherosclerotic, metabolic, and hypertensive diseases. Res Q Exerc Sport 66(4):268-275

15. Cooney GM, Dwan K, Greig CA, Lawlor DA, Rimer J, Waugh FR et al (2013) Exercise for depression. Cochrane Database Syst Rev 9:CD004366. doi:10. 1002/14651858.cd004366.pub6

16. Ekkekakis P(2015) Honey, Ishrunkthe pooled SMD! Guide to critical appraisal of systematic reviews and meta-analyses using the Cochrane review on exercise for depression as example. 2015;8:21-36. Ment Health Phys Act 8:21-36

17. Rimer J, Dwan K, Lawlor DA, Greig CA, McMurdo $M$, Morley W et al (2012) Exercise for depression. Cochrane Database Syst Rev 7:CD004366. doi:10. 1002/14651858.CD004366.pub5

18. Beck AT, Ward CH, Mendelson M, Mock J, Erbaugh $\mathrm{J}$ (1961) An inventory for measuring depression. Arch Gen Psychiatry 4:561-571

19. Hamilton M (1967) Development of a rating scale for primary depressive illness. Br J Soc Clin Psychol 6(4):278-296

20. Gabler H, Nitsch JR, Singer R (2001) Einführung in die Sportpsychologie. Teil 2: Anwendungsfelder. Verlag Karl Hofmann, Schorndorf

21. Stoll O, Ziemainz H (2012) Laufen psychotherapeutisch nutzen: Grundlagen, Praxis, Grenzen. Springer, Berlin Heidelberg

22. Dietrich A (2006) Transient hypofrontality as a mechanism for the psychological effects of exercise. Psychiatry Res 145(1):79-83 
23. Ide K, Secher NH (2000) Cerebral blood flow and metabolism during exercise. Prog Neurobiol 61(4):397-414

24. Dietrich A (2003) Functional neuroanatomy of altered states of consciousness: the transient hypofrontality hypothesis. Conscious Cogn 12(2):231-256

25. Duncan J, Owen AM (2000) Common regions of the human frontal lobe recruited by diverse cognitive demands. Trends Neurosci 23(10):475-483

26. D'Esposito M, Postle BR, Rypma B (2000) Prefrontal cortical contributions to working memory: evidence from event-related fMRI studies. Exp Brain Res 133(1):3-11

27. Dietrich A, Sparling PB (2004) Endurance exercise selectively impairs prefrontal-dependent cognition. Brain Cogn 55(3):516-524

28. Ekkekakis P (2009) The Dual-Mode Theory of affective responses to exercise in metatheoretical context: I. Initial impetus, basic postulates, and philosophical framework. Int Rev Sport Exerc Psychol 2(1):73-94

29. Ekkekakis P (2009) The Dual-Mode Theory of affective responses to exercise in metatheoretical context: II. Bodiless heads, ethereal cognitive schemata, and other improbable dualistic creatures, exercising. Int Rev Sport Exerc Psychol 2(2):139-160

30. Broocks A, Rieckmann P (2014) Sport und Neuroplastizität bei psychischen Erkrankungen. InfNeurol Psychiatr 16(10):51-59

31. Comer RJ (1995) Klinische Psychologie. Spektrum, Heidelberg Oxford

32. Blomstrand $E$, Hassmén $P$, Ekblom $B$, Newsholme EA (1991) Administration of branched-chain amino acids during sustained exercise-effects on performance and on plasma concentration of some amino acids. Eur J Appl Physiol Occup Physiol 63(2):83-88

33. ChaouloffF (1997) Effects of acute physical exercise on central serotonergic systems. Med Sci Sports Exerc 29(1):58-62

34. Broocks A (2000) Körperliche Aktivität und psychische Gesundheit: Psychische und neurobiologische Effekte von Ausdauertraining und Agoraphobie. Steinkopff, Darmstadt

35. Schmaal L, Veltman DJ, van Erp TGM, Samann PG, Frodl T, Jahanshad N et al (2016) Subcortical brain alterations in major depressive disorder: findings from the ENIGMA major depressive disorder working group. Mol Psychiatry 21(6):806-812

36. Pereira AC, Huddleston DE, Brickman AM, Sosunov AA, Hen R, McKhann GM et al (2007) An in vivo correlate of exercise-induced neurogenesis in the adult dentate gyrus. Proc Natl Acad Sci U S A 104(13):5638-5643

37. Knapen J, Sommerijns E, Vancampfort D, Sienaert P, Pieters G, Haake P et al (2009) State anxiety and subjective well-being responses to acute bouts of aerobic exercise in patients with depressive and anxiety disorders. Br J Sports Med 43(10):756-759

38. Ahmadler F (2007) Therapeutische Anwendung von Ausdauertraining bei Patienten mit verschiedenen psychischen Störungen. Untersuchung zur Änderungsmotivation und Besserung der Compliance. Dissertation, Universität Lübeck

39. Edenfield TM (2007) Exercise and mood: Exploring the role of exercise in regulating stress reactivity in bipolar disorder. Dissertation, University of Maine

40. Meyer JD, Koltyn KF, Stegner AJ, Kim J, Cook DB (2016) Influence of exercise intensity for improving depressed mood in depression: a dose-response study. Behav Ther 47(4):527-537
41. Busch AM, Ciccolo JT, Puspitasari AJ, Nosrat $S$, Whitworth JW, Stults-Kolehmainen M (2016) Preferences for exercise as a treatment for depression. Ment Health Phys Act 10:68-72

42. Kopp M (2009) Körperliche Aktivität bei Menschen mit schweren psychischen Erkrankungen: Stand der Forschung und praktische Empfehlungen. Neuropsychiatrie 23(3):151-156

43. Bridle C, Spanjers K, Patel S, Atherton NM, Lamb SE (2012) Effect of exercise on depression severity in older people: systematic review and meta-analysis of randomised controlled trials. Br J Psychiatry 201(3):180-185

44. Baxter $\mathrm{H}$, Winder $\mathrm{R}$, Chalder $\mathrm{M}$, Wright $\mathrm{C}$, Sherlock S, Haase A et al (2010) Physical activity as a treatment for depression: the TREAD randomised trial protocol. Trials 11:105

45. Chalder M, Wiles NJ, Campbell J, Hollinghurst SP, Haase AM, Taylor AH et al (2012) Facilitated physical activity as a treatment for depressed adults: randomised controlled trial. BMJ 344:e2758

46. Chalder M, Wiles NJ, Campbell J, Hollinghurst SP, Haase AM, Taylor AH et al (2013) Republished research:facilitated physical activity as a treatment for depressed adults: randomised controlled trial. BrJ Sports Med 47(10):629

47. Belvederi Murri $M$, Amore $M$, Menchetti $M$, Toni G, Neviani F, Cerri M et al (2015) Physical exercise for late-life major depression. Br J Psychiatry 207(3):235-242

48. Blumenthal JA, Babyak MA, Moore KA, Craighead WE, Herman S, Khatri $P$ et al (1999) Effects of exercise training on older patients with major depression. Arch Intern Med 159(19):2349-2356

49. Babyak M, Blumenthal JA, Herman S, Khatri $P$, Doraiswamy M, Moore $\mathrm{K}$ et al (2000) Exercise treatment for major depression: maintenance of therapeutic benefit at 10 months. Psychosom Med 62(5):633-638

50. Herman S, Blumenthal JA, Babyak M, Khatri $P$, Craighead WE, Krishnan KR et al (2002) Exercise therapy for depression in middle-aged and older adults: predictors of early dropout and treatment failure. Health Psychol 21(6):553-563

51. Blumenthal JA, Babyak MA, Doraiswamy PM, Watkins L, Hoffman BM, Barbour KA et al (2007) Exercise and pharmacotherapy in the treatment of major depressive disorder. Psychosom Med 69(7):587-596

52. Hoffman BM, BabyakMA, CraigheadWE, Sherwood A, Murali Doraiswamy P, Coons MJ et al (2011) Exercise and pharmacotherapy in patients with major depression: one-year follow-up of the SMILE study. Psychosom Med 73(2):127-133

53. Blumenthal JA, Babyak MA, O'Connor C, Keteyian S, Landzberg J, Howlett J et al (2012) Effects of exercise training on depressive symptoms in patients with chronic heart failure: the HF-ACTION randomized trial. JAMA 308(5):465-474

54. Brenes GA, Williamson JD, Messier SP, Rejeski WJ, Pahor M, Ip E et al (2007) Treatment of minor depression in older adults: a pilot study comparing sertraline and exercise. Aging Ment Health 11(1):61-68

55. Callaghan P, Khalil E, Morres I, Carter T (2011) Pragmatic randomised controlled trial of preferred intensity exercise in women living with depression. BMC Public Health 11:465

56. Chu I-H, Buckworth J, Kirby TE et al (2009) Effect of exercise intensity on depressive symptoms in women. Ment Health Phys Act 2(1):37-43

57. Craft LL, Perna FM (2004) The benefits of exercise for the clinically depressed. Prim Care Companion J Clin Psychiatry 6(3):104-111
58. Craft LL, Freund KM, Culpepper L, Perna FM (2007) Intervention study of exercise for depressive symptoms in women. J Womens Health (Larchmt) 16(10):1499-1509

59. Danielsson L, Papoulias I, Petersson E, Carlsson J, Waern M (2014) Exercise or basic body awareness therapy as add-on treatment for major depression: a controlled study. J Affect Disord 168:98-106

60. Doyne EJ, Ossip-Klein DJ, Bowman ED, Osborn KM, McDougall-Wilson IB, Neimeyer RA (1987) Running versus weight lifting in the treatment of depression.J Consult Clin Psychol 55(5):748-754

61. Ossip-Klein DJ, Doyne EJ, Bowman ED, Osborn KM, McDougall-Wilson IB, Neimeyer RA (1989) Effects of running or weight lifting on self-concept in clinically depressed women. J Consult Clin Psychol 57(1):158-161

62. Dunn AL, Trivedi MH, Kampert JB, Clark CG, Chambliss HO (2002) The DOSE study: a clinical trial to examine efficacy and dose response of exercise as treatment for depression. Control Clin Trials 23(5):584-603

63. Dunn AL, Trivedi MH, Kampert JB, Clark CG, Chambliss HO (2005) Exercise treatment for depression: efficacy and dose response. Am J Prev Med 28(1):1-8

64. Foley LS, Prapavessis H, Osuch EA et al (2008) An examination of potential mechanisms for exercise as a treatment for depression: apilot study. Ment Health Phys Act 1(2):69-73

65. Fremont JCLW (1987) Aerobic exercise and cognitive therapy in the treatment of dysphoric moods. Cognit Ther Res 11(2):241-251

66. Gary RA, Dunbar SB, Higgins MK, Musselman DL, Smith AL (2010) Combined exercise and cognitive behavioral therapy improves outcomes in patients with heart failure. JPsychosom Res 69(2):119-131

67. Hallgren M, Kraepelien M, Öjehagen A, Lindefors N, Zeebari Z, Kaldo V et al (2015) Physical exercise and internet-based cognitive-behavioural therapy in the treatment of depression: randomised controlled trial. Br J Psychiatry 207(3):227-234

68. Hemat-Far A, Shahsavari A, Roholla Mousavi S (2012) Effects of selected aerobic exercises on the depression and concentrations of plasma serotonin in the depressed female students aged 18 to 25. J Appl Res Clin Exp Ther 12(1):47

69. Ho CWH, Chan SC, Wong JS, Cheung WT, Chung DWS, Lau TFO (2014) Effect of aerobic exercise training on chinese population with mild to moderate depression in Hong Kong. Rehabil Res Pract 2014:627376

70. Hoffman JM, Bell KR, Powell JM, Behr J, Dunn EC, Dikmen Set al (2010) A randomized controlled tria of exercise to improve mood after traumatic brain injury. PMR 2(10):911-919

71. Wise EK, Hoffman JM, Powell JM, Bombardier CH, Bell KR (2012) Benefits of exercise maintenance after traumatic brain injury. Arch Phys Med Rehabi 93(8):1319-1323

72. Huang T, Liu C, Tsai Y, Chin Y, Wong C (2015) Physical fitness exercise versus cognitive behavior therapy on reducing the depressive symptoms among community-dwelling elderly adults: a randomized controlled trial. Int J Nurs Stud 52(10):1542-1552

73. Kerling A, Tegtbur U, Gützlaff E, Kück M, Borchert L, Ates Z et al (2015) Effects of adjunctive exercise on physiological and psychological parameters in depression: a randomized pilot trial. J Affect Disord 177:1-6

74. Kerse N, Hayman KJ, Moyes SA, Peri K, Robinson E, Dowell A etal (2010) Home-based activity program for older people with depressive symptoms: 
DeLLITE - a randomized controlled trial. Ann Fam Med 8(3):214-223

75. Klein MH, Greist JH, Gurman AS et al (1984) A comparative outcome study of group psychotherapy vs. exercise treatments for depression. Int J Ment Health 13(3-4):148-176

76. Krogh J, Saltin B, Gluud C et al (2009) The DEMO trial: a randomized, parallel-group, observerblinded clinical trial of strength versus aerobic versus relaxation training for patients with mild to moderate depression. J Clin Psychiatry 70(6):790-800

77. Krogh J, Videbech P, Thomsen C, Gluud C, Nordentoft M (2012) DEMO-II trial. Aerobic exercise versus stretching exercise in patients with major depression-a randomised clinical trial. PLOS ONE 7(10):e48316

78. Krogh J, Speyer H, Gluud C, Nordentoft M (2015) Exercise for patients with major depression: a protocol for a systematic review with metaanalysis and trial sequential analysis. Syst Rev 4:40

79. La de Cerda P, Cervelló E, Cocca A, Viciana J (2011) Effect of an aerobic training program as complementary therapy in patients with moderate depression. Percept Mot Skills 112(3):761-769

80. Lavretsky $\mathrm{H}$, Alstein LL, Olmstead RE, Ercoli LM, Riparetti-Brown M, Cyr NS et al (2011) Complementary use of tai chi chih augments escitalopram treatment of geriatric depression: a randomized controlled trial. Am J Geriatr Psychiatry 19(10):839-850

81. Legrand F, Heuze JP (2007) Antidepressant effects associated with different exercise conditions in participants with depression: a pilot study. J Sport ExercPsychol 29(3):348-364

82. Martinsen EW, Medhus A, Sandvik L (1985) Effects of aerobic exercise on depression: a controlled study. Br Med J (Clin Res Ed) 291(6488):109

83. Martiny K, Refsgaard E, Lund V, Lunde M, Sørensen L, Thougaard B et al (2012) A 9-week randomized trial comparing a chronotherapeutic intervention (wake and light therapy) to exercise in major depressive disorder patients treated with duloxetine. JClin Psychiatry 73(9):1234-1242

84. Mather AS, Rodriguez C, Guthrie MF, McHarg AM, Reid IC, McMurdo MET (2002) Effects of exercise on depressive symptoms in older adults with poorly responsive depressive disorder: randomised controlled trial. Br J Psychiatry 180:411-415

85. McCann IL, Holmes DS (1984) Influence of aerobic exercise on depression. J Pers Soc Psychol 46(5):1142-1147

86. McNeil JK, LeBlanc EM, Joyner M (1991) The effect of exercise on depressive symptoms in the moderately depressed elderly. Psychol Aging 6(3):487-488

87. Mota-Pereira J, Silverio J, Carvalho S, Ribeiro JC, Fonte D, Ramos J (2011) Moderate exercise improves depression parameters in treatmentresistant patients with major depressive disorder. JPsychiatr Res 45(8):1005-1011

88. Nabkasorn C, Miyai N, Sootmongkol A, Junprasert $\mathrm{S}$, Yamamoto $\mathrm{H}$, Arita M et al (2006) Effects of physical exercise on depression, neuroendocrine stress hormones and physiological fitness in adolescent females with depressive symptoms. Eur J Public Health 16(2):179-184

89. Pfaff JJ, Alfonso H, Newton RU, Sim M, Flicker L, Almeida OP (2014) ACTIVEDEP: a randomised, controlled trial of a home-based exercise intervention to alleviate depression in middle-aged and older adults. Br JSports Med 48(3):226-232

90. Pilu A, Sorba M, Hardoy MC, Floris AL, Mannu F, Seruis ML et al (2007) Efficacy of physical activity in the adjunctive treatment of major depressive disorders: preliminary results. Clin Pract Epidemiol Ment Health 3:8

91. Carta MG, Hardoy MC, Pilu A, Sorba M, Floris AL, Mannu FA et al (2008) Improving physical quality of life with group physical activity in the adjunctive treatment of major depressive disorder. Clin Pract Epidemiol Ment Health 4:1

92. Pinchasov BB, Shurgaja AM, Grischin OV, Putilov AA (2000) Mood and energy regulation in seasonal and non-seasonal depression before and after midday treatment with physical exercise or bright light. Psychiatry Res 94(1):29-42

93. Schuch FB, Vasconcelos-Moreno MP, Borowsky C, Fleck MP (2011) Exercise and severe depression: preliminary results of an add-on study. J Affect Disord 133(3):615-618

94. Shahidi M, Mojtahed A, Modabbernia A, Mojtahed M, Shafiabady A, Delavar A et al (2011) Laughter yoga versus group exercise program in elderly depressed women: a randomized controlled trial. Int J Geriatr Psychiatry 26(3):322-327

95. Sims J, Galea M, Taylor N, Dodd K, Jespersen $S$, Joubert $L$ et al (2009) Regenerate: assessing the feasibility of a strength-training program to enhance the physical and mental health of chronic post stroke patients with depression. Int J Geriatr Psychiatry 24(1):76-83

96. Teoh V, Sims J, Milgrom J (2009) Psychosocial predictors of quality of life in a sample of communitydwelling stroke survivors: a longitudinal study. Top Stroke Rehabil 16(2):157-166

97. Singh NA, Clements KM, Fiatarone MA (1997) A randomized controlled trial of progressive resistance training in depressed elders. J Gerontol A Biol Sci Med Sci 52(1):M27-35

98. Singh NA, Stavrinos TM, Scarbek Y, Galambos G, Liber C, Fiatarone Singh MA (2005) A randomized controlled trial of high versus low intensity weight training versus general practitioner care for clinical depression in older adults. JGerontol A Biol Sci Med Sci 60(6):768-776

99. Tsang HWH, Fung KMT, Chan ASM, Lee G, Chan F (2006) Effect of a qigong exercise programme on elderly with depression. Int J Geriatr Psychiatry 21(9):890-897

100. Veale D, Le Fevre K, Pantelis C, de Souza V, Mann A, Sargeant A (1992) Aerobic exercise in the adjunctive treatment of depression: a randomized controlled trial. J R Soc Med 85(9):541-544

101. Verrusio W, Andreozzi $P$, Marigliano B, Renzi A, Gianturco V, Pecci MT et al (2014) Exercise training and music therapy in elderly with depressive syndrome: a pilot study. Complement Ther Med 22(4):614-620

102. Williams CL, Tappen RM (2008) Exercise training for depressed older adults with Alzheimer's disease. Aging Ment Health 12(1):72-80

103. Zarshenas S, Houshvar P, Tahmasebi A (2013) The effect of short-term aerobic exercise on depression and body image in Iranian women. Depress Res Treat 2013:132684

\section{Wo steht Deutschland in puncto Gesundheitsverhalten im europäischen Vergleich?}

Die Bevölkerung in Deutschland ist sportlich aktiver als der europäische Durchschnitt. Beim Rauschtrinken allerdings ist der Anteil hierzulande sogar überdurchschnittlich hoch. Das ergaben neue Daten aus dem Robert Koch-Institut.

Im EU-weiten Vergleich liegt Deutschland beim täglichen Obstkonsum nur im hinteren Drittel. Beim Raucher-Anteil liegen die Werte in Deutschland relativ nah am EUDurchschnitt. Das sind Ergebnisse aus dem Monitoring zum Thema Gesundheitsverhalten in Deutschland und Europa.

Die Autoren aus dem Robert Koch-Institut (RKI) berichten über Ergebnisse der Studie Gesundheit in Deutschland aktuell (GEDA 2014/2015-EHIS) und stellen Vergleiche mit anderen EU-Staaten und zwischen den Bundesländern an. Die GEDA-Studie mit ihren mehr als 20.000 Teilnehmern ist Teil des Gesundheitsmonitorings, mit dem das RKI kontinuierlich Daten zur Gesundheit der Bevölkerung erhebt und analysiert. Mit GEDA 2014/2015-EHIS ist es erstmals möglich, Informationen zur Gesundheitssituation und zum Gesundheitsverhalten mit einheitlichen Methoden europaweit zu erheben und miteinander zu vergleichen

\section{Neu: Sport in Freizeit und Job}

Körperliche Aktivität ist Thema mehrerer Fact Sheets, sie umfasst mehr als nur Sport. Die Weltgesundheitsorganisation empfiehlt mindestens 150 Minuten mäßig anstrengende Ausdaueraktivität pro Woche. Im EU-Durchschnitt erreichen rund ein Viertel der Frauen und ein Drittel der Männer diese Empfehlung. In Deutschland schaffen knapp die Hälfte der Frauen und etwas mehr als die Hälfte der Männer das Ausdauerziel. In der aktuellen Ausgabe werden erstmals Daten zur körperlichen Aktivität in der Freizeit und zugleich zur körperlichen Aktivität bei der Arbeit vorgestellt.

Quelle: Robert Koch-Institut www.rki.de/journalhealthmonitoring 


\section{U. Rauchfleisch, O.F. Kernberg, P. Briken, B. Dulz (Hrsg.)}

\section{Handbuch der Antisozialen Persönlichkeitsstörung}

\section{Stuttgart: Schattauer Verlag 2017, 715 S., 15 Abb., (ISBN: 978-3-7945-3063-2), geb. 99.99 EUR}

Die Herausgeber dieses Werkes, das erstmals die heterogenen Aspekte antisozialen Verhaltens zum Gegenstand eines Handbuches macht, beginnen ihr Vorwort mit einer op-

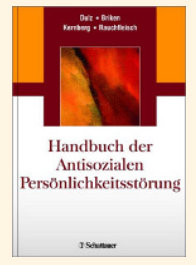
timistischen Vision Seit vor 20 Jahren Ärzte und Psychologen begonnen hätten, systematisch mit Borderline-Patienten zu arbeiten,

was bis dahin als kaum möglich galt, sei es nicht zuletzt durch die spezifischen Psychotherapieverfahren - dazu gekommen, dass die Prognose im Vergleich zu anderen psychischen Störungen gut sei. Eine ähnliche Erwartung ist offenbar mit der mit der Antisozialen Persönlichkeitsstörung (ASP) verbunden. Hier wird im Vorwort ebenfalls konstatiert, dass bei Anwendung spezifischer Psychotherapieverfahren die Prognose heute besser sei als bei vielen anderen psychischen Störungen. Dieses für forensisch Erfahrene etwas überraschende Diktum weckt Interesse und zeigt den hohen Anspruch der Herausgeber, die alle auf dem Felde der Persönlichkeitsstörungen ausgewiesene Experten sind.

Eine Schwierigkeit der ASP liegt in der Heterogenität des Konstruktes, das zwar griffig benannt, aber dennoch ideengeschichtlich und terminologisch recht vieldeutig ist. Diese Situation spiegelt sich in der breiten Themenpalette des Bandes mit 47 recht knapp gefassten Einzelbeiträgen, die sich der Geschichte und Epidemiologie, den Grundlagen, der Klassifikation und Diagnostik, der Symptomatologie und der Therapie widmen. Anschauliche Tabellen,prägnante Merksätze undinformative Fallbeispiele unterstützen in einigen Abschnitten didaktisch geschickt die Aufbereitung der enorm heterogenen Materie. Dabei werden auch sozialpolitisch interessante Randgebiete nicht ausgespart, etwa mit Themen wie White Collar Kriminalität oder des antisozialen Verhaltens der Mächtigen in Form von Wirtschafts-, Staats und Regierungskriminalität. Dies reicht bis zum anspruchsvollen Vorhaben einer "Psychopathologie der Leadership". Wieder aufgenommen wird auch das sozialpsychologische Konstrukt eines „antiautoritären Charakters”, das aus den gesellschaftspolitischen Umbrüchen der 68er Jahre geläufig ist.

In einem wichtigen Grundlagenkapitel über Genetik kommt Svenn Torgersen zum Fazit, das Risiko für Antisozialität steige nicht nur mit zunehmender genetischer Vulnerabilität, sondern auch mit einer zunehmend negativen Umwelt, wobei offen bleibe, inwieweit dies auf einer Gen-Umwelt-Korrelation beruhe. Die "Jagd“ nach einem antisozialen Gen wird jedenfalls skeptisch gesehen, da wir nie wissen könnten, inwieweit der Grad an Antisozialität auf Gene oder auf die Umwelt zurückzuführen sei. Eher ernüchternd sind auch die von Jürgen Müller vorgestellten Befunde zur Neurobiologie und Bildgebung der ASP, wobei insbesondere die Heterogenität des klinischen Konstrukts die Aussagekraft der oftmals inkonsistenten und kaum replizierten Ergebnisse einschränke. Dies wird auch beim interdisziplinären Kommunikationsprozess in einem juristischen Kontext zu berücksichtigen sein, wenn es um die Relevanz der neurobiologischen Befunde etwa für forensische Begutachtungsfragen geht. Hier hat vorerst und wohl auf gegenwärtig unabsehbare Zeit der Primat der psychopathologischen Analyse zu gelten. Dies zeigt sich auch im Kapitel von Kröber über die Beurteilung der Schuldfähigkeit von Persönlichkeitsstörungen einschließlich ihrer antisozialen Ausprägungen, wobei zu Recht noch einmal betont wird, dass für die Zuschreibung einer "schweren anderen seelischen Abartigkeit" im Sinne der §§ 20,21 StGB nicht primär die Diagnose, sondern die konkrete Ausgestaltung der vorliegenden Störung von Bedeutung ist.

An vielen Stellen des Buches zeigt sich eine Vorliebe der Herausgeber und ihrer Autoren für Konzepte der Psychoanalyse bzw. nachfolgender tiefenpsychologischer Schulen. Deutlich wird dies z.B. im Beitrag von Michael Stone über die "Dunkle Seite“ von antisozialer Persönlichkeit, Psychopathie (verstanden i.S. der Psychopathy sensu Hare) und malignem Narzissmus. Reichhaltig klinisch belegt sind die Ausführungen von Otto F. Kernberg zur Differentialdiagnose antisozialen Verhaltens. Interessante Einzelkapitel gelten klinisch wichtigen oder wissenschaftlichen Aspekten von Antisozialität bzw. Psychopathy, etwa den affektiven Phänomene von Spannung und Impulsivität, dem selbstverletzenden Verhalten, der Suizidalität und den dissoziativen Phänomene, die seit der frühen Beschreibung des Ganser-Syndroms immer wieder zu Debatten über die Bedeutung dissoziativer Bewusstseinszustände im zeitlichen Umfeld von Straftaten geführt haben.

Im Abschnitt über die Therapie überwiegen psychodynamisch orientierte Behandlungsansätze und Fortentwicklungen, etwa mit der mentalisierungsbasierten oder der Schematherapie. Vertreten sind aber auch stärker kognitiv-behavioral fundierte Verfahren, die ja im forensischen Maßregelvollzug wie in der allgemeinen Kriminaltherapie zunehmend dominieren. Auch auf wichtige Sonderfragen wie die Behandlungsmöglichkeiten in der sozialtherapeutischen Anstalt oder im Strafvollzug und die häufigen komorbiden Suchtstörungen wird eingegangen.

Eine besondere Bedeutung erhält aufgrund der optimistischen Eingangserwartungen der Herausgeber das Kapitel über die Analyse der Psychotherapieforschung. Das Fazit bleibt vorerst ernüchternd, da trotz zunehmender Aktivitäten die Forschungslage zur Effizienz der psychotherapeutischen Interventionen bei der ASP unbefriedigend ist. Für die Gruppe der psychodynamisch orientierten Therapien wurde bislang kein wissenschaftlicher Nachweis der Wirksamkeit gefunden. Wirksame Behandlungen seien solche, die sich an kognitiv-verhaltenstherapeutischen Prinzipien orientieren und strukturierte, belohnungsorientierte Interventionen anbieten. Hervorgehoben werden die in der Regel sehr schwierigen Bedingungen der Therapie von Personen mit antisozialer Persönlichkeitsstörung im Kontext geschlossener Institutionen. Die Kriterien für die Dissoziale bzw. Antisoziale Persönlichkeitsstörung gemäß ICD-10 und DSM-5 einschließlich des dortigen „Alternativen Modelles" sowie ein reichhaltiges Literaturverzeichnis runden diesen umfassenden Band ab und machen ihn zu einer interessanten Grundlage für weitere Bemühungen auf diesem für Theorie wie Praxis weiterhin recht steinigen Acker.

H. Saß (Aachen) 